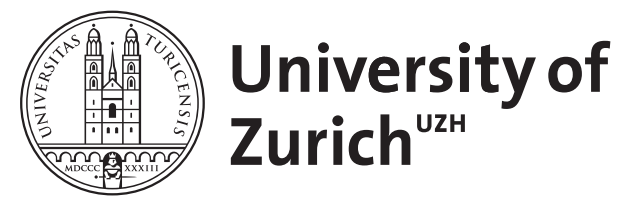

\title{
Collateral smile
}

Leippold, Markus ; Su, Lujing

\begin{abstract}
We analyze the impact of funding costs and margin requirements on index options traded on the CBOE. Assuming differential borrowing and lending rates, we derive no-arbitrage bounds for European options. We show that funding costs and the CBOE's margin requirements lead to a price increase, which translates into skew and smile patterns for implied volatility curves even under constant volatilities. Empirical tests confirm that our model-implied slopes have significant statistical power in explaining the slopes observed in the market. Hence, at least in part, funding costs and collateral requirements offer an institutional explanation of the volatility smile phenomenon.
\end{abstract}

DOI: https://doi.org/10.1016/j.jbankfin.2015.03.019

Posted at the Zurich Open Repository and Archive, University of Zurich ZORA URL: https://doi.org/10.5167/uzh-119587

Journal Article

Accepted Version

Originally published at:

Leippold, Markus; Su, Lujing (2015). Collateral smile. Journal of Banking and Finance, 58:15 - 28.

DOI: https://doi.org/10.1016/j.jbankfin.2015.03.019 


\title{
Collateral Smile *
}

\section{MARKus LeIPPOLD ${ }^{\dagger}$ AND LUJING SU S $^{\ddagger}$}

\begin{abstract}
We analyze the impact of funding costs and margin requirements on index options traded on the CBOE. Assuming differential borrowing and lending rates, we derive noarbitrage bounds for European options. We show that funding costs and the CBOE's margin requirements lead to a price increase, which translates into skew and smile patterns for implied volatility curves even under constant volatilities. Empirical tests confirm that our model-implied slopes have significant statistical power in explaining the slopes observed in the market. Hence, at least in part, funding costs and collateral requirements offer an institutional explanation of the volatility smile phenomenon.
\end{abstract}

Keywords: Collateral requirements, funding costs, volatility smile, option pricing. JEL classification: G01, G12, G13.

*For their helpful comments, we would like to thank Carol Alexander (the editor) and an anonymous referee, as well as Panayiotis Andreou, David Bates, Jonathan Berk, Tony Berrada, Josh Coval, Stephen Figlewski, Rajna Gibson, Lasse H. Pedersen, Adriano Rampini, Paul Whelan, Alexandre Ziegler, and the participants at the Revelstoke Finance Seminar, the Conference of the Swiss Society for Financial Market Research, the 18th Annual Conference of the Multinational Finance Society, Swiss Finance Institute Asset Pricing Workshop 2011, AFA 2013 San Diego, and the finance seminars at the University of Geneva and the University of Zurich for useful comments. Financial support by the Swiss Finance Institute (SFI), Bank Vontobel, and the National Center of Competence in Research "Financial Valuation and Risk Management" is gratefully acknowledged.

${ }^{\dagger}$ University of Zurich, Department of Banking and Finance, Plattenstrasse 14, 8032 Zurich, Switzerland; markus.leippold@bf.uzh.ch; +41 (44) 6345069.

$\ddagger$ University of Zurich, Department of Banking and Finance, Plattenstrasse 14, 8032 Zurich, Switzerland; lujing.su@bf.uzh.ch; +41 (44) 6343931. 


\section{Introduction}

We analyze the impact of funding costs and margin requirements on the price bounds of index options traded on the Chicago Board Options Exchange (CBOE). Margin requirements are collateral the option sellers are required to deposit with the exchange. Funding costs refer to the spread between borrowing and lending rates. We propose a model that gives upper and lower bounds for option prices in the absence of arbitrage in a dynamically incomplete market with differential borrowing and lending rates. We show that funding costs and margin requirements generate arbitrage bounds that allow for skew and smile patterns for implied volatilities that are consistent with what we typically observe in option markets. Empirical tests show that our model-implied slopes have significant statistical power in explaining the slopes observed in the market.

Imposing margin requirements or collateral requirements is common practice in both over the counter (OTC) and exchange-based transactions. During the recent financial crisis, market participants had to painfully acknowledge that the value of a derivative depends not only on its payoff structure, but also on the counterparty's creditworthiness. To mitigate counterparty risk, the contracting party for whom the derivative has a negative value is required to deposit collateral on a margin account to guarantee a certain recovery rate in case of default. While on OTC markets the use of collateral became widespread only over the past few years, standardized margin requirements have been used at exchanges already since the late 1980s. Yet, for the most part, the option pricing literature has been silent on how these margin requirements influence the pricing of exchange-traded derivatives. Therefore, we take a closer look at the price impact of collateral rules on exchange-traded index options.

For the pricing of options, the funding costs, measured as the spread between borrowing and lending rates, must also influence the replicating strategy. The money needed to purchase the underlying and to be deposited in the margin account is borrowed at a rate that exceeds 
the rate at which short selling proceeds can be invested. Indeed, assuming a significantly higher borrowing rate is in line with the currently prevailing market conditions. In the recent financial crisis, banks had difficulties in funding and maintaining a certain level of liquidity. These difficulties were further exacerbated by mutual distrust and an increasing reluctance to lend money to one another. Very quickly, interbank money markets dried out. In particular, cash lending became quite restricted and other key funding sources were also inaccessible.

A commonly agreed-upon measure of funding difficulty is the difference between the Libor and the Overnight Index Swap rate (OIS). Between 2002 and the beginning of the recent financial crisis, the three-month Libor-OIS spread was usually around 10 to 30 basis points (bps). However, it jumped to 66 bps on August 20, 2007, and remained high until March 2009, with a peak of 364 bps on October 10, 2008. In a situation in which the historically stable Libor-OIS spread varies dramatically and rises to new levels, the assumption of a single riskfree rate for borrowing and lending may no longer be appropriate. The wedge between these two rates in interplay with collateral requirements may then have an economically significant impact on the pricing of derivatives. Hence, we put forward a model which takes these two market frictions into account.

To isolate the effect of collateral requirements and funding costs on option prices, we choose the classical Black-Scholes model as our starting point. However, we work in an incomplete market framework as in Bergman (1995), which allows us to drive a wedge between the borrowing and lending rate. In an incomplete market, a unique equilibrium option price can only be derived when additional assumptions on the structure of the economy are made. Nevertheless, the absence of arbitrage allows us to put meaningful bounds on option prices. Hence, we extend the model of Bergman (1995) by incorporating collateral requirements and we derive solutions for the upper and lower bounds of option prices.

Depending on the margin rules, the exact form of the option upper prices varies for different exchanges. We investigate explicitly the impact of the margin requirements imposed 
by the CBOE. By choosing parameter values based on historical data, we show that this margin adjustment plays a non-negligible role in determining upper bounds of option prices. Furthermore, its relative importance varies with moneyness. We illustrate numerically that the option implied volatility bounds accounting for margin requirements and funding costs as imposed by the $\mathrm{CBOE}$ are capable of allowing for substantial volatility smiles, similar in magnitude to those observed in the data. This feature of our model does not rely on jumps or stochastic volatilities of the underlying price processes, which may already and in part explain the observed volatility smile. Hence, not only deviations from the geometric Brownian motion assumption, but also the general institutional set-up of the market may be responsible for a significant part of the observed implied volatility patterns.

Bringing our model to the data seems to be a promising next step. In particular, we challenge our constant volatility model by testing whether we could generate volatility slopes comparable with the empirical ones. Following the methodology applied in Bakshi, Kapadia, and Madan (2003), we find a clear link between the empirical slope and the slope predicted by our model. A simple ordinary least square regression (OLS) on the differences shows that, on average, our theoretical slope changes can already account for more than $30 \%$ of the time variation of the empirical slope changes. Therefore, our model provides an additional avenue to explain at least in part the variation of implied volatility smiles.

Taking margin and funding costs into account is not new in derivative pricing. For instance, Johannes and Sundaresan (2007) discuss the impact of collateral on swap prices. Using Eurodollar futures rates, they found that swaps are priced above the traditional portfolio of forwards value and below a portfolio of futures value. Berkovich and Shachmurove (2013) argue that the collateral requirement for a trading strategy is path dependent. Once the actual cost of implementing a put selling strategy is fully taken into account, writing put options on S\&P 500 index (SPX) earns only normal returns or even negative returns. Lou (2009) shows how the recently observed negative swap spread can be explained by asymmetric 
funding costs.

Our study is also related to papers that investigate option pricing bounds when the BlackScholes assumption of a dynamically complete and frictionless market is violated. In an incomplete market, the no-arbitrage argument alone is not enough to determine a unique option price. Instead, option prices must lie in a band that corresponds to the expected value of the option payoff under all the measures that rule out arbitrage. The papers closely related to our study are Santa-Clara and Saretto (2009), Bergman (1995) and Piterbarg (2010). However, our work differs from these papers in at least four ways.

Firstly, Santa-Clara and Saretto (2009) argue that the margin calls and funding costs could make the strategy involving selling OTM puts unprofitable, and thus OTM put options remain expensive. Our paper, however, studies directly the impact of these two market frictions on option prices.

Secondly, Bergman (1995) studies the impact of funding costs on option prices and derives the resulting no-arbitrage bounds. However, he does not consider the impact of collateral requirements at all, which may lead to some counterintuitive results when inverting the noarbitrage bounds for prices to no-arbitrage bounds for implied volatility. In particular, in the model of Bergman (1995) the upper no-arbitrage bound for put options degenerate to a constant. Hence, the existence of differential borrowing and lending rates cannot generate any smile pattern.

Thirdly, allowing for differential borrowing and lending rates is a complication that Piterbarg (2010) does not consider. Piterbarg (2010) introduces the intricacy of differential rates based on the types of assets that are used to secure the funding, but the same rate is used for borrowing and lending. In contrast, our paper looks at the impact of differential borrowing and lending rates on option prices. In addition, in Piterbarg (2010) the probability measure is implicitly fixed without further specification. Hence, there are unique option prices. However, in our model the analysis is based on no-arbitrage bounds, since the market is inherently 
imperfect due to the wedge between borrowing and lending rate. ${ }^{1}$

Fourthly, we provide evidence on the actual impact on option prices of the collateral rules as explicitly specified by the CBOE. Furthermore, using option price data, we also test the performance of our model by fitting empirical implied volatility curves. To our best knowledge, these aspects have not been considered by previous papers.

We organize the paper as follows. In Section 2, we provide an overview of the collateral requirements for options traded on the CBOE. In Section 3, we develop an option pricing model that accounts for funding costs and margin requirements. We derive the upper and lower bounds of option prices under the CBOE margin rules. In Section 4, weg bring our model to the data and conduct an empirical study of implied volatility slopes. Section 5 concludes.

\section{Margin requirements for derivatives in practice}

Collateral requirements on exchanges, usually referred to as margin requirements, are set by each exchange individually, and may differ across markets. For our analysis, we restrict ourselves to the world's largest option trading exchange, the CBOE. We explicitly focus on the margin requirements for the index options traded on the CBOE. In what follows, we briefly explain these margin requirements and we refer the interested reader to the CBOE's website for detailed explanations and specific examples. ${ }^{2}$

Margin requirements for buyers and sellers of options differ. Option buyers, who obtain a right rather than an obligation, are exempted from margin requirements once the full price of the option is paid. The reason is simple: buyers can always let the option expire without

\footnotetext{
${ }^{1}$ As an avenue of future research, it would be interesting to see how our model could be extended to narrow the pricing bounds or eventually obtain prices.

${ }^{2}$ The CBOE margin manual can be downloaded from http://www.cboe.com/tradtool/marginmanual2000.pdf. It provides a complete description of the margin requirements for the various option strategies.
} 
incurring further costs. Moreover, on the CBOE, for options with a time to expiration of more than nine months, buyers are allowed to pay $75 \%$ of the cost of the options as the initial margin with a maintenance margin at $75 \%$ of the option market value. In the following analysis, we assume that buyers pay the option price in full, since most liquid options have short maturities and, hence, need to be paid in full.

Writers of options are required to post margins to cover the risk of no delivery (when asked) at maturity. For example, writing a call option generates the risk of an unlimited loss, as the underlying price can increase to an arbitrarily large value. Therefore, call option sellers are required to deposit cash in the margin account to protect buyers against the sellers' default. The use of clearing houses guarantees that the option contract will be fulfilled. Therefore, we do not take into account the option writer's default risk in our model.

For option sellers, the CBOE uses two alternative margin rules, the strategy-based margin rules and the portfolio margining rules. Under the strategy-based margin rules, the positions are managed under the so-called strategy margin account and the margin is calculated according to each predefined option strategy. ${ }^{3}$ Strategy-based margin rules have been effective since the 1980s. In a private communication from the CBOE, we were informed that the strategy-based margin rules still remain effective for a significant part of the options traded on the CBOE. Therefore, we include these rules in our analysis.

Strategy-based margin rules use predefined formulas to compute margin requirements based on the strategy option writers apply. We let $M(t)$ denote the margin requirement for options. For a naked option traded on the CBOE, the strategy-based margin rule consists

\footnotetext{
${ }^{3}$ Examples of such strategies are, e.g., a short put, covered call, long vertical call spread, etc.. The CBOE provides a margin manual on its website to explain the details of the margin requirements for each type of strategy.
} 
of the option market value and some portion of the underlying value or strike price, and is

$$
\begin{array}{ll}
\text { Call: } & M_{c}(t)=\max \left(V(S, t)+a_{1} S(t)-(K-S(t))^{+}, V(S, t)+a_{2} S(t)\right), \\
\text { Put: } \quad M_{p}(t)=\max \left(V(S, t)+a_{1} S(t)-(S(t)-K)^{+}, V(S, t)+a_{2} K\right),
\end{array}
$$

where $M_{c}(t)$ and $M_{p}(t)$ are the margin amounts for call and put options respectively, $S(t)$ is the underlying price, $V(S, t)$ is the value of the option, and the parameters $a_{1}$ and $a_{2}$ represent the margin parameters specified by the CBOE. For options on a broad index, the CBOE currently sets the parameters $a_{1}$ and $a_{2}$ equal to 0.15 and 0.1 , respectively. For equity options or options written on a narrow based index, $a_{1}$ and $a_{2}$ are set equal to 0.2 and 0.15 . Note that these are the minimal margin requirements for strategy-based margin accounts for all types of investors, including brokers. Individual investors are sometimes subject to much higher margin parameters charged by the brokerage firms, which could reach $40 \%$ for $a_{1}$ and $35 \%$ for $a_{2}$ (Santa-Clara and Saretto (2009)).

On April 2, 2007, the CBOE amended the margin rules and introduced the portfolio margining rules, which allow charging margins based on the risk exposure of the whole option portfolio. For some positions, the margin requirements may not have changed significantly, but for positions with offsetting exposures, the difference can indeed be significant. The portfolio margining rule is a scenario-based rule that calculates the possible losses assuming various market moves. For SPX related products, the market moves in the underlying index are specified within a range of $-8 \%$ to $+6 \%$. The computed largest potential loss must then be compared with a per contract minimum of 37.50 dollars (for SPX options with multiplier 100). ${ }^{4}$ The greater of these two values defines the margin requirement. Currently, the option pricing model that the CBOE uses for computing the possible loss for option positions upon various market moves is not publicly available. Hence, the best thing we can do for our numerical analysis, is to assume that the CBOE uses the standard Black-Scholes formula to

\footnotetext{
${ }^{4}$ The per contract minimum is a fixed amount of cash and applies to SPX options with various strikes and maturities regardless of the underlying value. It has not been changed since the inception of the portfolio margin. Also note that at the CBOE, an option contract has a standardized multiplier of 100.
} 
determine portfolio margins.

We consider two types of portfolio margin requirements, namely the margin requirement for a naked short sale and the minimum margin requirement. The naked short sale portfolio requirement assumes there is only one option in the portfolio margin account, while the minimum portfolio margin requirement considers the least amount of capital that must be locked in the account for every option sold. Margin requirement is the amount of equity (cash) that must be maintained in a margin account. It is calculated as the sum of the market value of all long positions minus the sum of the market value of all short positions. Note that whenever an option is written in the portfolio, the cash balance generated by selling the option is usually kept in the account to offset the short position created by option writing. The margin for each option is therefore larger than the value of the option. The naked short-sell margin requirement under portfolio margin account, to be more specific, is

$$
\max _{k \in K}\{V((1+k) S, t), V(S, t)+37.50\}
$$

where $K=\{-0.08,-0.07, \ldots, 0.05,0.06\}$ is the set of market scenarios and $V((1+k) S, t)$ denotes the option price when the underlying moves from $S$ to $(1+k) S$ at time $t$. When the underlying value moves to $(1+k) S$, the loss generated from writing the option is $V((1+k) S, t)-$ $V(S, t)$. Adding up with the proceeds from option writing $V(S, t)$ yields $V((1+k) S, t)$. The margin requirement is thus the greater of the worst possible loss and the per contract minimum. For a naked short sale, as there is only one option in the account, it is straightforward that

$$
\begin{aligned}
& \text { Call: } \quad M_{c}(t)=\max \{V(1.06 S, t), V(S, t)+37.50\}, \\
& \text { Put: } \quad M_{p}(t)=\max \{V(0.92 S, t), V(S, t)+37.50\} .
\end{aligned}
$$

Realistically, investors hold not only one, but many options in their trading account. Hence, we must also analyze the margin requirement for writing an option when the investor is holding already a portfolio involving many options. This margin requirement depends on the loss and profit on the composition of the corresponding portfolio. Due to the lack of data on typical 
portfolios held at the CBOE, we circumvent this problem as follows. Instead of considering arbitrary portfolio compositions, we consider only the minimum margin requirement that writing an option incurs. In particular, the least possible margin requirement for a short option position in the portfolio is simply the sum of the per contract minimum and the option proceeds, i.e.,

$$
M_{c}(t)=V(S, t)+37.50, \quad M_{p}(t)=V(S, t)+37.50
$$

Note that this minimum portfolio margin is the least possible margin for any type of strategy. ${ }^{5}$ By using this minimum requirement, we get a conservative estimate of the margin's impact under the portfolio margining rules.

In the subsequent analysis, we use the three types of margining rules, i.e., the strategybased margin rule for a naked short sale in equation (1), the portfolio margining rule for a naked short sale in (3), and the minimum possible portfolio margining rule for writing an option in (4).

\section{Option pricing with costly margin requirements}

In terms of the price dynamics, we base our analysis on the standard Black-Scholes assumptions. However, we depart from the Black-Scholes model by introducing differential borrowing and lending rates as well as margin requirements for option writers. As Bergman (1995) argues, a dynamically incomplete capital market allows the existence of a wedge between borrowing and lending rates. Depending on the structure of the market, equilibrium option prices may depend on other state variables. Even though a pure no-arbitrage argument cannot uniquely determine option prices, we can derive option pricing bounds, the violation of which indicates arbitrage opportunities even after accounting for market imperfections.

\footnotetext{
${ }^{5}$ For example, for a covered call strategy, the option seller also needs to satisfy the minimum portfolio requirement.
} 
To analyze the option pricing problem with differential borrowing and lending rates, we introduce three accounts. The first is a cash account, where cash is deposited to finance the purchase of the underlying and to hold the proceeds from short selling the underlying. It plays the role of a traditional savings account where the deposited cash earns the lending rate $r_{l}$ and borrowing is not allowed. Our second account is a debt account, from which the option writer can borrow the funds used for the replicating portfolio if the writer's cash holding is not enough. The debt account is charged at the borrowing rate $r_{b}$. The third account is the collateral account to secure the margin requirement. The deposited cash earns the lending rate $r_{l}$. To simplify computations, we assume that the borrowing and lending rates are constant. In general, we have $r_{b} \geq r_{l}$, as the spread between the two rates reflects the return the bank must earn for its operations.

\subsection{No-arbitrage bounds}

Within our incomplete market setting, we cannot derive a unique option price unless we impose some additional structure. However, arbitrage considerations help us to derive pricing bounds on the options. To obtain these bounds, we need to analyze the portfolio strategy that replicates the payoff of the option at expiration. The replicating strategy in our case is defined by a four-dimensional process $(\alpha(t), \beta(t), \lambda(t), \delta(t))$ to capture the different interest rates earned on different accounts. By $\alpha(t)$, we denote the amount of stocks that we hold at

time $t$; by $\beta(t)<0$, the cash borrowed from the debt account; by $\lambda(t)>0$, the cash deposited at the cash account; and by $\delta(t)$, the cash deposited in the collateral account.

To prevent arbitrage, we can show that the option price $V(S, t)$ with a payoff of $h(S(T))$ at expiration $T \geq t \geq 0$ must lie within an upper and a lower bound. The underlying price is denoted by $S(t)$ with a continuous dividend yield $r_{d}$. We first focus on the lower bound, and 
consider the following minimization problem:

$$
\mathcal{M}^{-} \quad: \quad \min _{\alpha(t), \beta(t), \lambda(t), \delta(t)} V(S, 0)
$$

subject to

$$
\begin{aligned}
V(S, t) & =\alpha(t) S(t)+\beta(t)+\lambda(t)+\delta(t) \\
d V(S, t) & =\alpha(t)\left(d S(t)+r_{d} S(t) d t\right)+r_{b} \beta(t) d t+r_{l} \lambda(t) d t+r_{l} \delta(t) d t \\
V(S, T) & \geq-h(S(T)) \\
\delta(t) & \geq M(t) .
\end{aligned}
$$

The constraints for the $\mathcal{M}^{-}$-problem can be interpreted as follows. The first constraint is the value dynamics of the replicating portfolio, the second constraint is the self-financing condition, and the third constraint guarantees that the payoff of the replicating strategy is at least as large as the contractual payoff of the contingent claim. Finally, $\delta(t) \geq M(t)$ represents the collateral requirements during the lifetime of the contract. The solution to the $\mathcal{M}^{-}$-problem, we denote it by $V_{0}^{-}$, is the strategy out of all eligible strategies satisfying these constraints which costs the minimum dollar amount at initiation of the contract. Hence, $-V_{0}^{-}$ is the minimal price an option buyer is willing to pay. As discussed in Section 2, the collateral requirements for option buyers are zero. Therefore, $\delta(t)$ is zero in the optimal solution and collateral does not play a role in determining $V_{0}^{-}$.

Since an investment used to replicate a non-positive payoff must have a non-positive initial capital, $V_{0}^{-}$is less than or equal to zero. Furthermore, if $V<-V_{0}^{-}$we could buy the option and implement the strategy that solves the $\mathcal{M}^{-}$-problem. Therefore, $V \geq-V_{0}^{-}$must hold. This strategy meets the collateral requirement for the option buyer and gives a payoff that is greater than $-h(S(T))$. The combined payoff thus gives a non-negative payoff at maturity and generates a positive cashflow at the initial time, $-V_{0}^{-}-V>0$. Since the collateral requirements for option buyers are zero, as discussed in Section $2, \delta(t)$ is zero in the optimal 
solution. Therefore, collateral does not play a role in determining $V_{0}^{-}$.

To determine the upper arbitrage bounds, we consider the following optimization problem,

$$
\mathcal{M}^{+}: \min _{\alpha(t), \beta(t), \lambda(t), \delta(t)} V(S, 0),
$$

subject to

$$
\begin{aligned}
V(S, t) & =\alpha(t) S(t)+\beta(t)+\lambda(t)+\delta(t), \\
d V(S, t) & =\alpha(t)\left(d S(t)+r_{d} S(t) d t\right)+r_{b} \beta(t) d t+r_{l} \lambda(t) d t+r_{l} \delta(t) d t, \\
V(S, T) & \geq+h(S(T)), \\
\delta(t) & \geq M(t) .
\end{aligned}
$$

We denote the solution to the $\mathcal{M}^{+}$-problem by $V_{0}^{+} . V \leq V_{0}^{+}$has to hold if arbitrage opportunities are to be excluded. When $V>V_{0}^{+}$, selling the option and employing the strategy that solves the $\mathcal{M}^{+}$-problem is an arbitrage opportunity. This strategy satisfies the collateral requirement for option writers and gives a payoff greater than $+h(S(T))$. Therefore, the combining strategy has a non-negative payoff at maturity and generates a positive cashflow at initiation, i.e., $V-V_{0}^{+}>0$. We can now summarize the above discussion in the following proposition.

Proposition 1. In a dynamically incomplete market with $r_{l} \neq r_{b}$ and with collateral requirements, the option price $V_{0}^{e}$ must lie within the arbitrage band $\left[-V_{0}^{-}, V_{0}^{+}\right]$, where $V_{0}^{-}$and $V_{0}^{+}$ solve $\mathcal{M}^{-}$and $\mathcal{M}^{+}$, respectively.

As collateral has no impact on the lower bound of option prices, the lower bound corresponds exactly to the one derived by Bergman (1995), who also considers differential borrowing and lending rates. Therefore, we borrow the following result: ${ }^{6}$

\footnotetext{
${ }^{6}$ We do not repeat the derivation here, but refer to Bergman (1995) for details.
} 
Proposition 2 (Bergman (1995)). In the Black-Scholes setting, but under differential borrowing and lending rates, the lower bound for calls is given by the classical Black-Scholes call option formula with the lending rate replacing the risk-free rate. For put options, the risk-free rate is replaced by the borrowing rate.

However, for the determination of the upper bounds, i.e., the solution to $\mathcal{M}^{+}$, we cannot rely on Bergman (1995), as he does not take collateral into account.

\subsection{General formulas for upper price bounds}

In the Black-Scholes model, borrowing or lending occurs at the same interest rate. Therefore, the same PDE applies for the pricing of both puts and calls, but with different boundary conditions. However, in the presence of funding costs, the replicating strategy for puts and calls involves different positions in the cash, debt, and collateral accounts. This leads to subtle differences in the PDE representation of calls and puts. In the case of a call option, we must carefully segregate the positions into i) a collateral $M_{c}(t)$ required by the exchange to be deposited in the cash account earning the lending rate, $i i)$ the quantity $V(S, t)-M_{c}(t)$ borrowed at the borrowing rate from the debt account to finance the posting of margin, and finally iii) $\alpha(t) S(t)$ borrowed from the debt account to finance the stock purchase. ${ }^{7}$

In the case of a put option, we have to track separately the positions in the cash, debt, and collateral accounts by decomposing them as above into i) the collateral $M_{p}(t)$ deposited in the cash account, ii) the quantity $V(S, t)-M_{p}(t)$ borrowed to finance the required margin, and iii) the short selling proceeds $\alpha(t) S(t)$ deposited in the cash account. The put option's price is not sufficient to meet the margin requirement and $V(S, t)-M_{p}(t)$ needs to be funded by borrowing. The relative size of $\alpha(t) S(t)$ and $V(S, t)-M_{p}(t)$ is not known. Thus we assume that the short selling proceeds $\alpha(t) S(t)$ are saved in the cash account and cannot be used to

\footnotetext{
${ }^{7}$ Even under the portfolio-based margin rule, the proceeds of selling options must be kept in the margin account. Therefore $V(S, t)-M_{c}(t)$ is indeed borrowing.
} 
satisfy the margin requirement. This assumption not only simplifies the model, but is also consistent with market practice. Short sellers are generally required to leave the short sale proceeds in an interest bearing account with their broker until the short position is closed. We summarize the resulting pricing formulas below. The proof is given in Appendix A.

Proposition 3. In the Black-Scholes setting, but under differential borrowing and lending rates, the upper bound for call options in the presence of collateral requirements is given by the expectation

$$
V_{c}(S, t)=\mathbb{E}_{t}^{\mathbb{P}_{b}}\left[e^{-r_{b}(T-t)} V_{c}(S, T)+\int_{t}^{T} e^{-r_{b}(u-t)}\left(r_{b}-r_{l}\right) M_{c}(u) d u\right]
$$

under the pricing measure $\mathbb{P}_{b}$, subject to $V_{c}(S, T)=(S(T)-K)^{+}$and

$$
d S(t) / S(t)=\left(r_{b}-r_{d}\right) d t+\sigma d W^{b}(t)
$$

where $W^{b}(t)$ is a standard Brownian motion under $\mathbb{P}^{b}$. Under the pricing measure $\mathbb{P}_{b}$, the stock price discounted by the interest rate differential $r_{b}-r_{l}$ is a martingale. The corresponding upper bound for put options is given by the expectation

$$
V_{p}(t)=\mathbb{E}_{t}^{\mathbb{P}_{l}}\left[e^{-r_{b}(T-t)} V_{p}(S, T)+\int_{t}^{T} e^{-r_{b}(u-t)}\left(r_{b}-r_{l}\right) M_{p}(u) d u\right]
$$

under the pricing measure $\mathbb{P}_{l}$, subject to $V_{p}(S, T)=(K-S(T))^{+}$and

$$
d S(t) / S(t)=\left(r_{l}-r_{d}\right) d t+\sigma d W^{l}(t)
$$

where $W^{l}(t)$ is a standard Brownian motion under $\mathbb{P}_{l}$. Under the pricing measure $\mathbb{P}_{l}$, the stock price discounted by the interest rate differential $r_{l}-r_{d}$ is a martingale.

Intuitively, the pricing formulas in the proposition have two components. For instance, in the case of the call option, the first component $\mathbb{E}_{t}^{\mathbb{P}_{b}}\left[e^{-r_{b}(T-t)} V(S, T)\right]$ plays the role of the traditional Black-Scholes call option price, but with a different probability measure and discount rate. The second part, $\mathbb{E}_{t}^{\mathbb{P}_{b}}\left[\int_{t}^{T} e^{-r_{b}(u-t)}\left(r_{b}-r_{l}\right) M(u) d u\right]$, reflects the impact of the 
margin requirements on the option price, and we refer to it as the margin adjustment. Since $M(t)>0$, the margin adjustment is always positive. We can interpret it as the additional price the writer requires to be compensated for the increasing replication cost induced by fulfilling the margin requirements. If it is costless to post collateral, i.e., if the collateral earns the same rate as the borrowing rate $r_{b}=r_{l}$, then the margin adjustment disappears and the margin requirement would not influence the call price at all. Indeed, when $r_{b}=r_{l}$, equations (7) and (8) collapse to the standard Black-Scholes formula. However, whenever $r_{b}>r_{l}$, which is usually the case, the margin requirements increase the replicating cost and the call option prices through the margin adjustment.

It is worth noting that Proposition 3 provides a general formula to compute upper bounds on option prices under margin constraints and funding costs. Even though we focus on SPX options traded on the CBOE, its application is not restricted to this particular case.

In our model, the difference of the upper bounds between put and call options not only lies in the boundary conditions, but also in the probability measures. Two probability measures arise in the upper bounds because the replication strategies for call and put options vary. Replicating call options involves buying the underlying stock using the borrowing rate. Replicating put options requires shorting the underlying stock and earning the lending rate. The interest rate required for the replication strategy determines the drift of $S(t)$ under the pricing measure. Thus, the drift is determined by $r_{b}$ for calls and by $r_{l}$ for puts. This subtle difference of the replicating strategy of call and put options results in different impacts of funding costs on call and put options with a higher impact observed for call options.

\subsection{Upper price bounds under the CBOE's margin requirement}

Having derived the general option pricing formula in the presence of funding costs and general margin requirements, we can now insert the specific margin rule of the CBOE into the pricing 
formula to obtain the upper bound under the actual margin rules. We consider for our analysis three margin requirements: the strategy margin requirement for a naked short sale, the portfolio margin requirement for a naked short sale, and the minimum portfolio margin requirement. We collect the corresponding formulas in the corollaries below, which follow directly from Proposition 3 and are proven in Appendix B.

For options subject to the CBOE's strategy margin requirements, the upper bound for a short sale can be derived in closed form as shown in the following corollary.

Corollary 1. At time $t$, the upper price bound for European call options subject to CBOE's strategy margin rules for a short sale with maturity $T$, strike $K$ is

$$
\begin{aligned}
V_{c}(S, t)= & S(t) e^{\left(r_{b}-r_{d}-r_{l}\right)(T-t)} N\left(d_{1}(T, t)\right)-K e^{-r_{l}(T-t)} N\left(d_{2}(T, t)\right) \\
& +\left(r_{b}-r_{l}\right) S(t) \int_{t}^{T} e^{\left(r_{b}-r_{d}-r_{l}\right)(u-t)}\left(a_{2} N\left(-d_{1}^{*}(u, t)\right)+a_{1} N\left(d_{1}(u, t)\right)\right) d u \\
& +\left(1+a_{1}\right)\left(r_{b}-r_{l}\right) S(t) \int_{t}^{T} e^{\left(r_{b}-r_{d}-r_{l}\right)(u-t)}\left(N\left(d_{1}^{*}(u, t)\right)-N\left(d_{1}(u, t)\right)\right) d u \\
& -\left(r_{b}-r_{l}\right) K \int_{t}^{T} e^{-r_{l}(u-t)}\left(N\left(d_{2}^{*}(u, t)\right)-N\left(d_{2}(u, t)\right)\right) d u
\end{aligned}
$$

with

$$
\begin{aligned}
& d_{1}(u, t)=\frac{\ln \left(\frac{S(t)}{K}\right)+\left(r_{b}-r_{d}+\frac{1}{2} \sigma^{2}\right)(u-t)}{\sigma \sqrt{u-t}}, \quad d_{2}(u, t)=d_{1}(u, t)-\sigma \sqrt{u-t}, \\
& d_{1}^{*}(u, t)=\frac{\ln \left(\frac{S(t)\left(1+a_{1}-a_{2}\right)}{K}\right)+\left(r_{b}-r_{d}+\frac{1}{2} \sigma^{2}\right)(u-t)}{\sigma \sqrt{u-t}}, \quad d_{2}^{*}(u, t)=d_{1}^{*}(u, t)-\sigma \sqrt{u-t},
\end{aligned}
$$

where $N(\cdot)$ denotes the standard normal cumulative distribution function.

Although these pricing formulas are somewhat lengthy, they are merely the sum of the classical Black-Scholes price and the margin adjustment term. Analogously, we can derive the upper bound for the put option value.

Corollary 2. At time $t$, the upper price bound for European put options subject to CBOE's 
strategy margin rules for a naked short sale with maturity $T$, strike $K$ is

$$
\begin{aligned}
V_{p}(S, t)= & K e^{-r_{l}(T-t)} N\left(-d_{2}(T, t)\right)-S(t) e^{-r_{d}(T-t)} N\left(-d_{1}(T, t)\right) \\
& +a_{2}\left(r_{b}-r_{l}\right) K \int_{t}^{T} e^{-r_{l}(u-t)}\left(N\left(-d_{2}^{*}(u, t)\right)+N\left(d_{2}^{* *}(u, t)\right)\right) d u \\
& +a_{1}\left(r_{b}-r_{l}\right) S(t) \int_{t}^{T} e^{-r_{d}(u-t)}\left(N\left(-d_{1}(u, t)\right)-N\left(-d_{1}^{*}(u, t)\right)\right) d u \\
& +\left(r_{b}-r_{l}\right) K \int_{t}^{T} e^{-r_{l}(u-t)}\left(N\left(-d_{2}^{* *}(u, t)\right)-N\left(-d_{2}(u, t)\right)\right) d u \\
& +\left(a_{1}-1\right)\left(r_{b}-r_{l}\right) S(t) \int_{t}^{T} e^{-r_{d}(u-t)}\left(N\left(-d_{1}^{* *}(u, t)\right)-N\left(-d_{1}(u, t)\right)\right) d u,
\end{aligned}
$$

with

$$
\begin{aligned}
d_{1}(u, t) & =\frac{\ln \left(\frac{S(t)}{K}\right)+\left(r_{l}-r_{d}+\frac{1}{2} \sigma^{2}\right)(u-t)}{\sigma \sqrt{u-t}}, \quad d_{2}(u, t)=d_{1}(u, t)-\sigma \sqrt{u-t} \\
d_{1}^{*}(u, t) & =\frac{\ln \left(\frac{a_{1} S(t)}{a_{2} K}\right)+\left(r_{l}-r_{d}+\frac{1}{2} \sigma^{2}\right)(u-t)}{\sigma \sqrt{u-t}}, \quad d_{2}^{*}(u, t)=d_{1}^{*}(u, t)-\sigma \sqrt{u-t}, \\
d_{1}^{* *}(u, t) & =\frac{\ln \left(\frac{\left(1-a_{1}\right) S(t)}{\left(1-a_{2}\right) K}\right)+\left(r_{l}-r_{d}+\frac{1}{2} \sigma^{2}\right)(u-t)}{\sigma \sqrt{u-t}}, \quad d_{2}^{* *}(u, t)=d_{1}^{* *}(u, t)-\sigma \sqrt{u-t}, .
\end{aligned}
$$

For the portfolio margin for a naked short sale, the margin requirements depend on the option pricing model, as the loss of the naked short sale is determined by the option value under various market moves. Therefore, we have to solve iteratively for the final option value by using standard numerical methods. However, for the minimum portfolio margin requirement, we can obtain a closed-form solution for option prices, since the margin requirement is the sum of a constant and the option value. For European call options, we get the following result.

Corollary 3. At time $t$, the upper price bound for a European call option with maturity $T$, strike $K$, and subject to CBOE's portfolio margining rule is

$$
V_{c}(S, t)=S(t) e^{\left(r_{b}-r_{d}-r_{l}\right)(T-t)} N\left(d_{1}(T, t)\right)-K e^{-r_{l}(T-t)} N\left(d_{2}(T, t)\right)+\mathrm{MA}\left(M_{c}, t\right),
$$

with $d_{1}(u, t)$ and $d_{2}(u, t)$ given in equation (9). MA denotes the margin adjustment part of the option prices. For the portfolio margin for a naked short sale, the margin adjustment MA 
$i s$

$$
\mathrm{MA}=\mathbb{E}_{t}^{\mathbb{P}_{b}}\left[\int_{t}^{T} e^{-r_{l}(u-t)}\left(r_{b}-r_{l}\right)\left(M_{c}(u)-V_{c}(S, u)\right) d u\right]
$$

where $M_{c}(u)=\max \left\{V_{c}(1.06 S, u), V_{c}(S, u)+37.50\right\}$. For the minimum portfolio margin, MA can be computed in closed-form:

$$
\mathrm{MA}=\frac{37.5\left(r_{b}-r_{l}\right)}{r_{l}}\left(1-e^{-r_{l}(T-t)}\right)
$$

Similarly, we can calculate the upper price bound for European put options under the portfolio margin rule.

Corollary 4. At time $t$, the upper price bound for a European put option with maturity $T$, strike $K$, and subject to CBOE's portfolio margining rule is

$$
V_{p}(S, t)=K e^{-r_{l}(T-t)} N\left(-d_{2}(T, t)\right)-S(t) e^{-r_{d}(T-t)} N\left(-d_{1}(T, t)\right)+\mathrm{MA}\left(M_{p}, t\right),
$$

with $d_{1}(u, t)$ and $d_{2}(u, t)$ given in equation (10). MA denotes the margin adjustment part of the option prices. For the portfolio margin for a naked short sale, the margin adjustment MA is

$$
\mathrm{MA}=\mathbb{E}_{t}^{\mathbb{P}_{l}}\left[\int_{t}^{T} e^{-r_{l}(u-t)}\left(r_{b}-r_{l}\right)\left(M_{p}(u)-V_{p}(S, u)\right) d u\right]
$$

where $M_{p}(u)=\max \left\{V_{p}(0.92 S, u), V_{p}(S, u)+37.50\right\}$. For the minimum portfolio margin, MA can be computed in closed-form:

$$
\mathrm{MA}=\frac{37.5\left(r_{b}-r_{l}\right)}{r_{l}}\left(1-e^{-r_{l}(T-t)}\right)
$$

\subsection{Numerical illustration}

To provide some intuition about the magnitude of the impact of funding costs and margin rules, we investigate the potential impact of these market frictions on the implied volatility 
surface. The implied volatilities are calculated using the classical Black-Scholes model for which we use the lending rate as interest rate. In Figure 1, we first plot the implied volatility (IV) curves when we take into account only funding costs but no margin requirements as in Bergman (1995). ${ }^{8}$ Excluding margin requirements, we can observe a smile for call options. But for put options, the upper bound is exactly the benchmark Black-Scholes price. Thus, the upper bound degenerates to a constant. Hence, in a setting with constant volatility and funding costs, but without collateral requirements as in Bergman (1995), there is no way to explain the typical smile pattern observed for put options.

The above results do not depend on the numerical values, but hold in general. To see this, we consider, e.g., the lower bound for call options. We recall that in the Bergman model this is given by plugging in the lending rate $r_{l}$ into the classical Black-Scholes formula. Since we use Black-Scholes with $r_{l}$ to convert prices into volatilities as benchmark, we get a constant upper bound corresponding to the volatility $\sigma$. For the upper bound for call options, we use $r_{b}>r_{l}$. Since the call option's sensitivity to interest rates is positive, using $r_{b}$ leads to higher call prices. Inverting these prices with our reference Black-Scholes model, we get therefore higher implied volatilities. Since for calls the interest rate sensitivity increases with increasing spot price, the prices of options with low $K / S$ ratios increases more, which leads to the pattern that we observe in Panel A of Figure 1. Recalling that the interest rate sensitivity for puts is negative, a similar line of arguments explains the pattern that we observe in Panel B of Figure 1 for put options.

Figure 2 plots the IV bounds for different maturities taking into account margin requirements. Panel A, C, and E in Figure 2 show the call options' IV bounds under the three different CBOE margin rules. Irrespective of moneyness and maturity, the lower IV bound degenerates to a constant corresponding to the standard Black-Scholes IV using the lend-

\footnotetext{
${ }^{8}$ As parameter values for our numerical illustration, we use the average three-month Overnight Index Swap rate (OIS rate) as proxy for the lending rate over the period January 2002 to August 2010. The corresponding average three-month US-dollar Libor rate is used as the borrowing rate. For the volatility parameter, we take the average of VIX as the input for the volatility.
} 


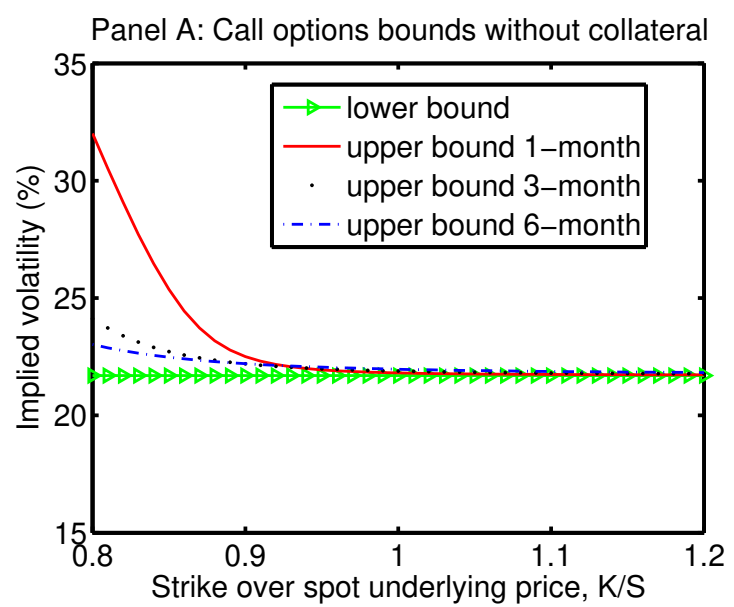

Panel B: Put options bounds without collateral

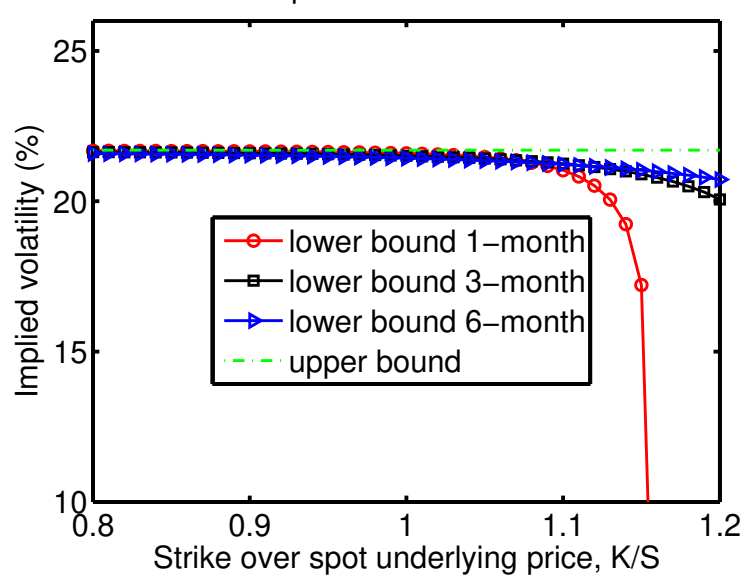

Figure 1: No-arbitrage bounds for implied volatility (IV) curves in the Bergman model. We plot the upper and lower bounds of the IV for options without margin requirements as predicted by Bergman (1995). Parameters are fixed as $r_{b}=0.026, r_{l}=0.023, \sigma=0.217$. We plot IV curves for calls in the left column and puts in the right column.

ing rate, our benchmark Black-Scholes price. The upper IV bound for calls is a decreasing function of strike for ITM options. For short-dated options, the implied volatility starts to increase again when the option turns OTM. ${ }^{9}$ Furthermore, the bounds are steepest for short maturities and gradually flatten out as maturity increases. Comparing the IV curves for the three margin rules, we find only small difference. The reason is that for call options, the price increase due to replicating strategy involving buying is much more pronounced unless call options go deeply OTM.

For put options, the IV bounds exhibit a different pattern. In Panel B, D, and F of Figure 2, we plot the IV bounds for put options. The lower bound for puts is the Black-Scholes price using the borrowing rate. Hence, these lower bounds are below the classical Black-Scholes price when the lending rate is used and equivalent to those in Figure 1, Panel B. However, for the upper bounds we observe an IV smile, which gradually steepens as the maturity decreases. This effect seems to be sensitive to the margin rules applied. The slope accounting for the strategy-based margin is the steepest, while the two types of portfolio margin rules generate

\footnotetext{
${ }^{9}$ This effect is most pronounced for the strategy margin requirement in Panel A. If we would extend the plot for deeper OTM options, this pattern would also be visible for the other margin requirements.
} 
Panel A: Call options bounds for strategy margin short sale

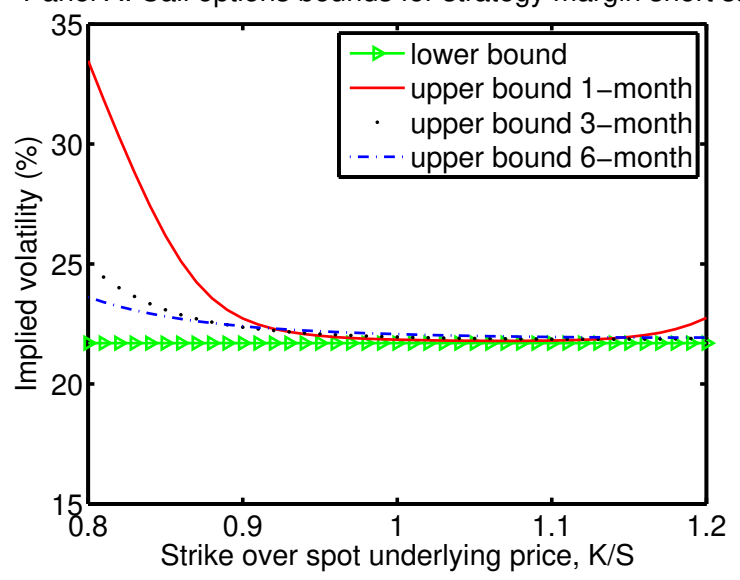

Panel C: Call options bounds for portfolio margin short sale

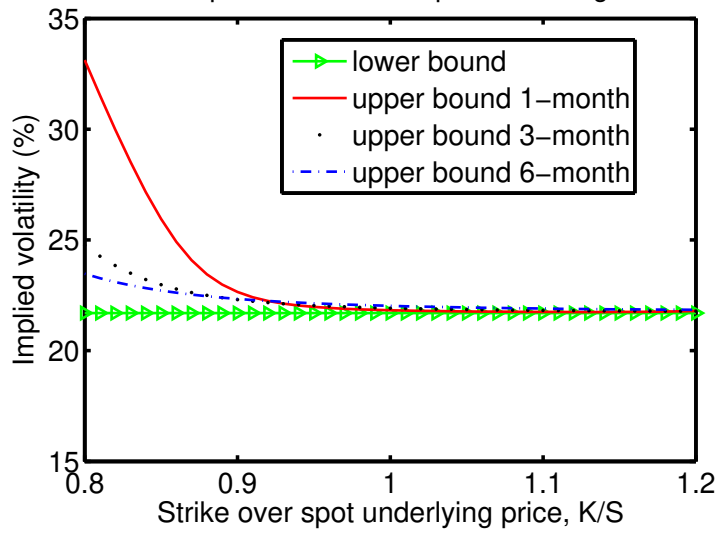

Panel E: Call options bounds for minimum portfolio margin

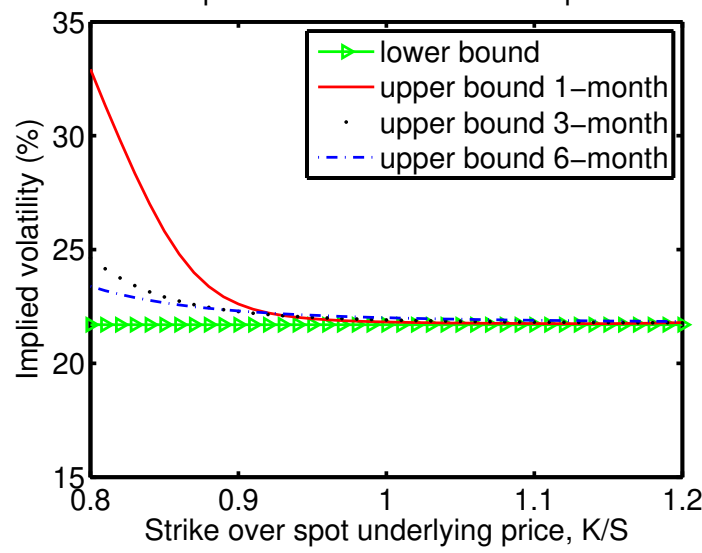

Panel B: Put options bounds for strategy margin short sale

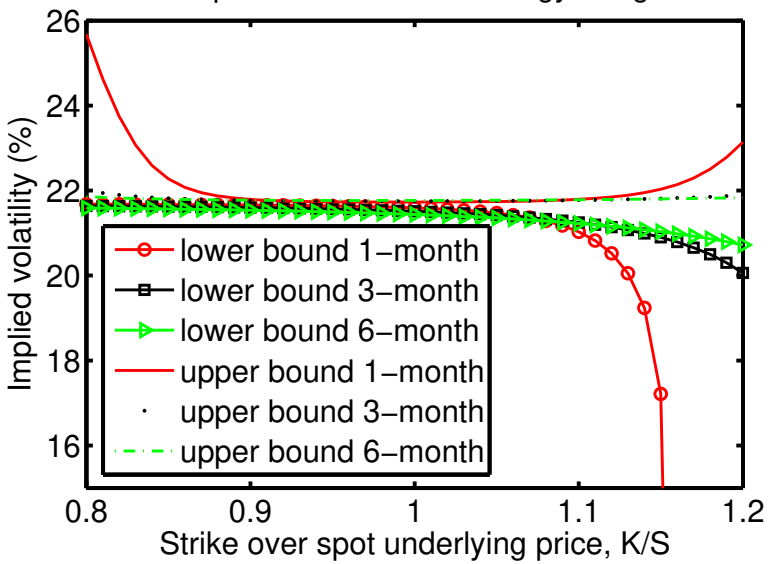

Panel D: Put options bounds for portfolio margin short sale

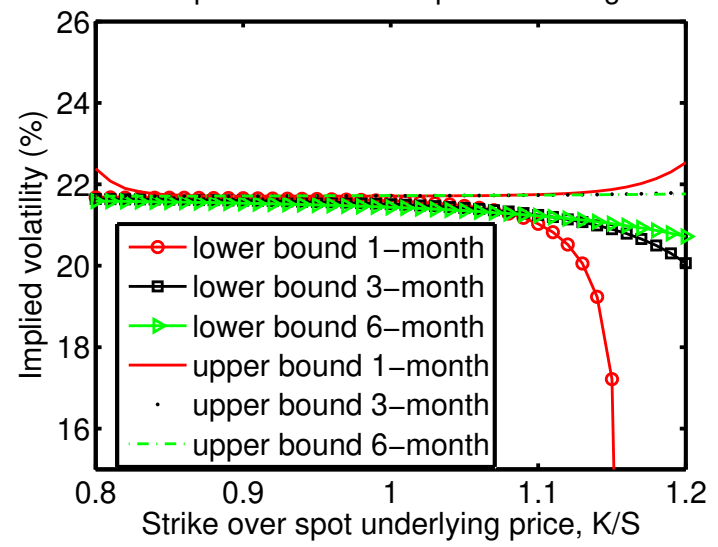

Panel F: Put options bounds for minimum portfolio margin

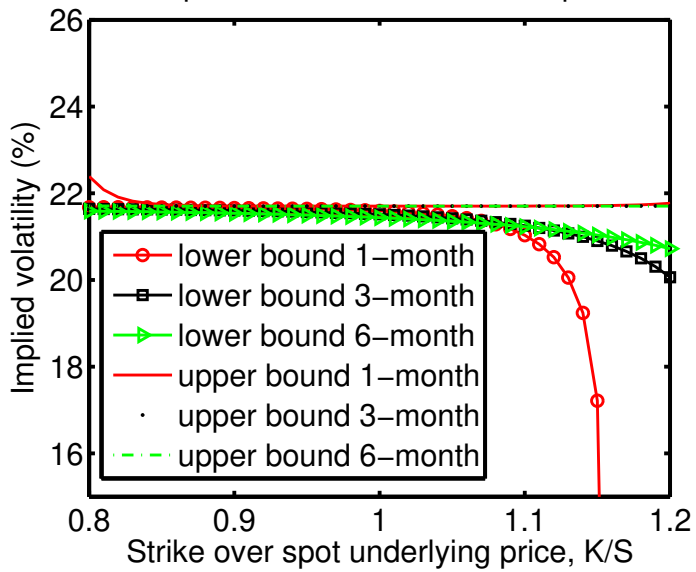

Figure 2: No-arbitrage bounds for implied volatility (IV) curves.

We plot the upper and lower bounds of the IV for options with one-month, three-month and sixmonth maturities for strategy-based margin requirements for a naked short sale in equation (1), the portfolio margining requirements for a naked short sale in (3) and minimum portfolio margining requirements in (4). Parameters are fixed as $r_{b}=0.026, r_{l}=0.023, \sigma=0.217, a_{1}=0.15, a_{2}=0.1$. We plot IV curves for calls in the left column and puts in the right column. 
similar smile patterns. Furthermore, we observe that the impact of funding costs on the upper bound of the IV surface is less pronounced for puts than for calls. Compared to a call, less borrowing is involved in replicating a put. Even though for both types of options the amount $V(S, t)-M(t)$ is borrowed from the debt account, the strategies on the underlying are different. For calls, investors borrow to purchase the underlying. For put options, investors profit from selling the underlying short. Therefore, funding costs increase the replicating cost of calls to a greater extent than for put options.

At least qualitatively, the above properties hold in general. To see this, e.g., in the case of the upper bounds, we can argue as follows. In the absence of margin requirements, we observe in Figure 1 that the upper bound for the put option is just the Black-Scholes IV. Adding margins gives a price increase, which naturally translates into higher Black-Scholes implied volatilities. To explain the particular pattern we observe, e.g., for put options in Figure 2, we can focus on the simplest case, which is the minimum collateral requirement given in Corollary 4. If we denote the fixed cash amount of 37.5 as imposed by the CBOE as a variable, say $q$, we can express the change in the Black-Scholes implied volatility as follows:

$$
\frac{\partial \sigma_{I V}}{\partial q}=\frac{\partial \sigma_{I V}}{\partial V_{p}(S, t)} \frac{\partial V_{p}(S, t)}{\partial q} .
$$

The first term on the right-hand side of (11) is the inverse of the option's vega, i.e., the sensitivity to volatility changes. For both puts and calls, the vega has a bell shape and is positive with the highest values around at-the-money options. Hence, the inverse obtains its highest values for out-of-the money and in-the-money options. The second term on the righthand side of (11) measures the increase in the option price for an increase in the cash-margin. Clearly, this quantity obtains high values for out-of-the money options. Putting these two effects together helps to justify the patterns as displayed in Figure 2.

We recall that the plots in Figure 2 represent upper and lower bounds. Hence, our results do not suggest that collateral requirements will indeed lead to more valued call options. We 
would have to add more structure to the model to provide sharper bounds. However, such extensions are beyond the scope of the present paper. Nevertheless, it is worth mentioning that Bollen and Whaley (2004) find that there is more demand for OTM index put options than OTM index call options. Consequently, market makers are likely to have larger net short positions in OTM put options. As portfolio margin rule uses as collateral the highest possible loss for the whole portfolio, selling put options is likely to put more collateral constraints on market makers. In contrast, selling call options might incur only the minimum collateral requirement. Therefore, the impact of collateral on OTM put options could be higher than on call options leading to higher IV of put options. Moreover, as put-call parity does not necessarily hold in the empirical data (see Kamara and Miller (1995) for example), we do not expect call and put options with the same strike to have exactly the same IV. ${ }^{10}$

\section{Empirical application}

To find empirical evidence on whether funding costs and collateral requirements have an impact on option prices, we analyze our model's ability to explain implied volatility slopes. If our model could add additional explanatory power to the factors previously used in the finance literature, our finding would provide a strong argument to incorporate funding costs and differential interest rates in option pricing models.

\footnotetext{
${ }^{10}$ Applying put-call parity by recognizing the effect of margin requirements and funding costs gives another prices bound. For example, given the model-implied call price bound, we can use put-call parity to obtain another put option price bound. The intersection of the put option price bound derived from the model and put-call parity gives a new bound. Numerical results show that this bound is wider than the options' own model-implied price bound. Therefore, put-call parity does not imply a sharper bound. The reason is that under funding costs and margin requirements, put-call parity generates two different inequalities.
} 


\subsection{Data processing}

We use data for options written on SPX from Ivy DB OptionMetrics for the sample period ranging from January 2002 to August $2010 .{ }^{11}$ Our data covers the period of the financial crisis. The high funding costs during this period provide ideal data points for our test. End-of-day bid and ask quotes, open-interest, volume, exercise price, IV, delta, gamma, dividend yield, and expiration dates on every call and put option are all provided by OptionMetrics.

Several filtering rules have been applied to obtain a clean data set. Firstly, we eliminated options with maturities less than eight days or more than 150 days to exclude any liquidityrelated bias. Secondly, we included only options with a positive trading volume, positive open interest, and positive bid prices. Finally, mid quotes lower than 0.375 , bid-ask spreads more than 1.5 times the mid-quotes, and strike over spot prices less than 0.7 or more than 1.3 were also excluded. This data contains in total 153,926 calls and 198,775 puts.

The lending rate is proxied by the US OIS rate and the US interbank borrowing rate is captured by the Libor rate. The interest rates are obtained from Bloomberg. ${ }^{12}$ To obtain the interest rates at different maturities we use linear interpolation.

As an alternative, we could also use the put-call parity to back out the borrowing and lending rate from options on SPX as was done, e.g., in Brenner and Galai (1996), Jackwerth and Rubinstein (1996), and Constantinides, Jackwerth, and Perrakis (2009), among others. However, in untabulated results, we find that our analysis and findings do not change and are robust to different specifications of the interest rates. As a further model input, we require a proxy for volatility, for which we use the VIX. ${ }^{13}$

\footnotetext{
${ }^{11}$ We focus on options on the SPX, as they are European options. Exchange-traded single stock options are of the American type and, hence, would complicate our analysis.

${ }^{12}$ Note that even though OptionMetrics provides index put options prices traded from 1996, the OIS rate from Bloomberg is only available since the end of 2001. Therefore, we select the data sample from 2002. Furthermore, using other proxies for the lending rate such as US Treasury rates produces similar results. The US Government began issuing four-week Treasury bills since mid 2001. Therefore, using the Treasury rate as the lending rate would not significantly extend our sample period.

${ }^{13}$ Initially, we considered three distinct volatility measures: 30-day historical volatility, VIX, and the IV
} 
As our model predicts different shapes for the put and call IV curve, we estimate the empirical slopes for puts and calls separately. When computing the IV, the midpoint of the best closing bid price and best closing offer price for the option is used. Following the standard methodology of Bakshi, Kapadia, and Madan (2003) (BKM hereafter), we derive the slope estimates weekly by pooling all the IV data in any given week (449 weeks) from Wednesday to Tuesday. We then sort the options according to their time-to-maturity into two groups, shortterm options (maturity of less than 60 days) and medium-term options (maturity between 60 and 150 days).

In addition to analyzing the whole sample period from January 2002 to August 2010, we also perform our tests on two subperiods including the pre-crisis period January 2002 to July 2007 and the crisis period August 2007 to July 2009. The results for these subperiods are similar to the whole sample. Therefore, they are omitted for brevity but can be obtained from the authors.

\subsection{Regression results for IV slopes}

To derive the slope estimate, different measures for the slope of the IV curve have been suggested in the literature. Here, we follow BKM (2003). They estimate the slope coefficient $\Pi$ using the regression equation

$$
\ln \left(\sigma^{i v}\left(y_{j}\right)\right)=\Pi_{0}+\Pi \ln \left(y_{j}\right)+\varepsilon_{j}, \quad j=1, \ldots, J
$$

where $y$ denotes the moneyness $K / S, \sigma^{i v}$ denotes the Black-Scholes IV and $J$ is the number of options available in the week. We perform the regression in (12) for each maturity group of call and put options to obtain weekly slope estimates.

Table 1 reports the estimated slope coefficients and the corresponding $R^{2}$ for each option

of the closest ATM 1-month options. The key results are robust and remain unchanged for these different measures. 
category. The slopes are negative and more pronounced for short-term options with slightly more negative slopes for calls than for puts. For each option group, the regression in equation (12) captures between 70-90\% of the variation in the IV slope. The slope for call options seems to be more negative than for put options, a result which is due to log transformation in regression (12). Indeed, not taking the logarithm in (12) or using other definition of slopes such as, e.g., in Han (2008), gives more negative slopes for puts. ${ }^{14}$ The empirical slopes we obtained show a strong persistence over time. Running augmented Dickey-Fuller test and choosing the number of lags by the Akaike information criterion suggests that slopes for all categories are non-stationary I(1) processes.

[Table 1 about here]

To obtain our model-implied slope $\Pi^{\text {model }}$ under constant volatility, we first compute the upper bounds of put and call options at nine different equally-spaced moneyness levels $K / S$ ranging from 0.8 to 1.2. We convert these prices to Black-Scholes IVs, which we then use for running the regression in equation (12). We use three types of margin rules to obtain the option upper bound and derive model-implied slopes. The following analysis is conducted for slopes derived using three margin rules.

To avoid the problem of spurious regression, we take the differences of all variables for the regression. Firstly, we run the $\Delta \Pi_{t}$ on its lag $\Delta \Pi_{t-1}$ as follows

$$
\Delta \Pi_{t}=\beta_{0}+\beta_{1} \Delta \Pi_{t-1}+\varepsilon_{t} .
$$

To see whether our model-implied slopes could explain the time variation of the empirical slopes, we regress the empirical slope change on the model-implied slope change

$$
\Delta \Pi_{t}=\beta_{0}+\beta_{1} \Delta \Pi_{t}^{\text {model }}+\beta_{2} \Delta \Pi_{t-1}+\varepsilon_{t},
$$

\footnotetext{
${ }^{14}$ In unreported regressions, we also used the slope definition from Han (2008), where the slope is measured as the negative of the average OTM put IV over the average ATM put and call options IV. The results are similar to what we find using the BKM (2003) slope definition.
} 
where the lagged slope difference $\Delta \Pi_{t-1}$ is included in the regression to correct for the autocorrelation in the dependent variable. We present the results from regressions (13) and (14) in Table 2 for different option groups.

[Table 2 about here]

Table 2 gives us several interesting findings. The lagged empirical slope difference, although always significant, can only explain a small portion of the evolution of $\Delta \Pi_{t}$ with average $R^{2}$ around 5 percent. For regression (14), we find that the coefficient for $\Delta \Pi_{t}^{\text {model }}$ for all margin rules is always positive and significant at the $1 \%$ level, indicating a positive link between the empirical slope difference and our model-implied slope difference. The coefficients for the put options are larger than those for the call options. This observation is in line with the findings of our numerical investigation in Section 3.4, where we find a steeper IV curve for calls as they are more sensitive to funding costs. The coefficient for calls does not differ much for different margin rules, which is again consistent with the finding in Section 3.4 that similar smiles are observed for call options.

For puts, however, we do observe quite different coefficients. As shown in our numerical analysis in Section 3.4, strategy-based margins tend to generate a steeper IV smile. Therefore, the coefficient is relatively small for strategy-based margins. Moreover, the coefficient for the minimum portfolio margins is also small compared to the portfolio margin for a naked short sale. The minimum portfolio margin tends to increase OTM IVs much more than ITM IVs, as the per contract minimum is substantial only for OTM options. In contrast, the naked short sale portfolio margin rules raise the IV of options across all moneyness levels, giving rise to a flatter smile. Therefore, the coefficient for the naked short sale portfolio margining is much higher than for the other two margin rules.

Finally, we see that for both puts and calls our model-implied slope can generate adjusted $R^{2}$-values around 23.6 percent for short-term options and around 39.1 percent for medium- 
term options. These findings provide evidence that our model helps to explain a substantial part of the time variations of empirical IV slopes differences.

\subsection{Regression results including control variables}

To compare the performance of regression (14) with those of other models, we also provide a regression analysis including other control variables. As a first set of control variables we consider the risk-neutral skewness and kurtosis. As shown by BKM (2003), the second and third moments of risk neutral distribution of returns have significant explanatory power in describing the time variation of empirical slopes.

As a second set of control variables, we consider the following three commonly used variables. We include the VIX as a proxy for market volatility, the previous six-month returns to capture stock market momentum, and a relative demand factor to control for demand impact. $^{15}$ In addition, since our model implies that funding costs matter for the slope of IV curves, we also include Libor-OIS spreads in our regression.

We start with the following specification of the regression equation based on risk-neutral parameters:

$$
\Delta \Pi_{t}=\beta_{0}+\beta_{1} \Delta \text { Skewness }_{t}+\beta_{2} \Delta \text { Kurtosis }_{t}+\beta_{3} \Delta \Pi_{t-1}+\varepsilon_{t} .
$$

As an additional exercise, we combine our model-implied slopes with risk-neutral parameters in one single regression as follows:

$$
\Delta \Pi_{t}=\beta_{0}+\beta_{1} \Delta \text { Skewness }_{t}+\beta_{2} \Delta \text { Kurtosis }_{t}+\beta_{3} \Delta \Pi_{t}^{\text {model }}+\beta_{4} \Delta \Pi_{t-1}+\varepsilon_{t} .
$$

\footnotetext{
${ }^{15}$ These variables are used to explain the time variations of the slope of the IV curves by, e.g., Amin, Coval, and Seyhun (2004), Li and Pearson (2005), Bollen and Whaley (2004) and Garleanu, Pedersen, and Poteshman (2009). Unfortunately, we do not have access to the data to measure the demand impact of end users as in Garleanu, Pedersen, and Poteshman (2009). We follow Han (2008) to measure the demand impact by the ratio of total open interest for OTM index put options (defined by $-\frac{3}{8}<\Delta_{P} \leq-\frac{1}{8}$ where $\Delta_{P}$ is the delta of put options ) to that for near and ATM index options (defined as call options with $\frac{3}{8}<\Delta_{C} \leq \frac{5}{8}$ and put options with $-\frac{1}{8}<\Delta_{P} \leq-\frac{3}{8}$ where $\Delta_{C}$ denotes the delta of call options).
} 
We run this regression for all of the three types of margin rules discussed in Section 2. We report the results for regressions (15) and (16) in Table 3.

[Table 3 about here]

For regression (15), we observe in Table 3 that the risk-neutral skewness is not significant at the 5 percent level for any option group. The risk neutral kurtosis becomes significant for the medium-term option group only. The lagged slope difference is always significant at any reasonable statistical level. However, using risk neutral factors alone gives quite low $R^{2}$ values. In the combined regression (16), we observe that the model-implied slope differences are significant at the 1 percent level for all types of margin rules and all option groups. The risk neutral factors remain insignificant for all option groups. The adjusted $R^{2}$ values have improved considerably by adding model-implied slope differences.

For the second set of control variables, we first run the following regression with control variables only,

$$
\begin{aligned}
\Delta \Pi_{t}=\beta_{0}+\beta_{1} \Delta \text { LiborOIS }_{t} & +\beta_{2} \Delta \operatorname{VIX}_{t}+\beta_{3} \Delta \text { IndexReturn }_{t} \\
& +\beta_{4} \Delta \text { RelativeDemand } \\
& +\beta_{5} \Delta \Pi_{t-1}+\varepsilon_{t} .
\end{aligned}
$$

Analogously, we also run a combined regression as follows,

$$
\begin{aligned}
\Delta \Pi_{t}=\beta_{0}+\beta_{1} \Delta \text { LiborOIS }_{t}+\beta_{2} \Delta \text { VIX }_{t}+\beta_{3} \Delta \text { IndexReturn }_{t} & \\
& \quad+\beta_{4} \Delta \text { RelativeDemand } \\
&
\end{aligned}
$$

We report the results for regression (17) and regression (18) in Table 4 for different options groups.

[Table 4 about here]

Referring to Table 4, we find that in regression (17), $\Delta \mathrm{VIX}_{t}$ is significant at the 5 percent level for all option groups. The demand factor is only significant for short-term calls. All other 
control variables are not significant at the 5 percent level. In the combined regression (18), $\Delta$ LiborOIS $_{t}$ and $\Delta$ VIX $_{t}$ are not always significant. Their coefficients switch signs for different option groups. However, the significance of $\Delta \Pi_{t}^{\text {model }}$ remains at the 1 percent level, even after

controlling for other variables. $\Delta \Pi_{t}^{\text {model }}$ changes from one week to the next because LiborOIS $_{t}$ and VIX change. As a non-linear function of LiborOIS ${ }_{t}$ and VIX, changes in $\Pi_{t}^{\text {model }}$ have additional power beyond that provided directly by changes in LiborOIS $t$ and $\mathrm{VIX}_{t}$. Indeed, when we include the model-implied slopes, we can substantially increase the explanatory power. The residual effect of our model-implied slope after controlling for VIX and the LiborOIS spread is positive, indicating that a higher implied slope change is followed by a higher empirical slope change. We remark that the above results are invariant to different margin requirements and hold for all option groups.

\section{Conclusion}

We presented a tractable option pricing model that accounts for margin requirements on exchanges and market participants' funding costs. In a dynamically incomplete market with differential rates, we derived upper and lower bounds for option prices with margin requirements when the underlying follows a geometric Brownian motion. Since margin requirements are positive, the prices derived from the upper bounds exceed the classical Black-Scholes option prices. For the margin rules of the world's most important option exchange, the CBOE, we derived upper price bounds for European call and put options. The relative difference between these upper bounds and the original Black-Scholes option prices turns out to be substantial, even under normal market conditions. Analyzing the funding costs in volatility space, the no-arbitrage region we obtained for the IV provides enough flexibility to allow volatility smiles and skews that are comparable in size to the empirically observed IV patterns. Consistent with empirical findings, the IV curve flattens out as the maturity increases. Hence, 
funding costs and collateral requirements offer an institutional explanation of the volatility smile phenomenon without departing from the constant volatility assumption.

The complexity of stock price processes and the variety of factors influencing option markets makes an empirical test of our model a delicate task. However, our model highlights that the slopes generated by the IV upper bounds under constant volatility assumption capture important factors in the time variation of the empirical slope change. By fitting the change of SPX option IV slopes, we found that our model-implied slopes are quite successful in explaining the empirical slopes, with average adjusted $R^{2}$ around 30 percent. The performance of our model-implied slope was compared with two regressions where risk-neutral factors and other commonly used variables are taken as the regressors. Using our model-implied slopes, we found that our institutional factors generate a level of adjusted $R^{2}$ much higher than the one generated by the commonly used factors. Furthermore, we ran a combined regression where both the model-implied slope and control variables are included. The regression results showed that our model-implied slopes remain significant and add explanatory power to the regression. Therefore, we conclude that our model, albeit simple, offers promising avenue for rationalizing the impact of margin requirements and funding costs on option prices.

\section{References}

Amin, K., J. D. Coval, and H. N. Seyhun, 2004, Index option prices and stock market momentum, Journal of Business 77, 835-874.

Bakshi, G., N. Kapadia, and D. Madan, 2003, Stock returns characteristics, skew laws, and the differential pricing of individual equity options, Review of Financial Studies 16, 101-143.

Bergman, Y., 1995, Option pricing with differential interest rates, Review of Financial Studies $53,475-500$. 
Berkovich, E., and Y. Shachmurove, 2013, An error of collateral: Why selling SPX put options may not be profitable, The Journal of Derivatives 20, 31-42.

Bollen, N. P. B., and E. R. Whaley, 2004, Does net buying pressure affect the shape of implied volatility functions?, Journal of Finance 59, 711-753.

Brenner, M., and D. Galai, 1996, Implied interest rates, Journal of Business 59, 493-509.

Constantinides, G. M., J. C. Jackwerth, and S. Perrakis, 2009, Mispricing of S\&P 500 index options, Review of Financial Studies 22, 1247-1277.

Garleanu, N., L. H. Pedersen, and A. Poteshman, 2009, Demand-based option pricing, Review of Financial Studies 22, 4259-4299.

Han, B., 2008, Investor sentiment and option prices, Review of Financial Studies 21, 387-414.

Jackwerth, J. C., and M. Rubinstein, 1996, Recovering probability distributions from option prices, Journal of Finance 51, 1611-1631.

Johannes, M., and S. Sundaresan, 2007, Pricing collateralized swaps, Journal of Finance 62, $383-410$.

Kamara, A., and T. W. Miller, 1995, Daily and intradaily tests of European put-call parity, Journal of Financial and Quantitative Analysis 30, 519-539.

Li, M., and N. Pearson, 2005, Price deviations of S\&P 500 index options from the BlackScholes formula follow a simple pattern, AFA 2006 Boston Meetings Paper.

Lou, W., 2009, On asymmetric funding of swaps and derivatives - a funding cost explanation of negative swap spreads, SSRN Working paper.

Piterbarg, V., 2010, Funding beyond discounting: Collateral agreements and derivatives pricing, Risk 2, 97-102. 
Santa-Clara, P., and A. Saretto, 2009, Option strategies: Good deals and margin calls, Journal of Financial Markets 12, 391-417. 


\section{Appendix}

\section{A Derivation of the upper price bounds}

We first derive the upper bounds for call options. We assume that the underlying price $S(t)$ follows a geometric Brownian motion with log-increments having constant volatility $\sigma$. Let $V(S, t)$ denote the upper bound of the derivative contract price. Applying Ito's lemma allows us to find the dynamics of $V(S, t)$ :

$$
d V(S, t)=\left(V_{t}^{\prime}(S, t)+\frac{1}{2} \sigma^{2} S^{2}(t) V_{s s}^{\prime \prime}(S, t)\right) d t+\alpha(t) d S(t),
$$

where $\alpha(t)=V_{s}^{\prime}(t)$. The option writer can construct a self-financing portfolio by holding $\alpha(t)$ units of stocks and taking positions in the debt, cash, and collateral accounts. We denote the corresponding amounts in these accounts by $\beta(t), \lambda(t)$, and $\delta(t)$. Hence, the replicating strategy has a value $U(S, t)=\alpha(t) S(t)+\beta(t)+\lambda(t)+\delta(t)$, which should be equal to $V(S, t)$. As self-financing implies no injection of external capital, the dynamics of the hedging portfolio must be

$$
d U(S, t)=\alpha(t)\left(d S(t)+r_{d} S(t) d t\right)+r_{b} \beta(t) d t+r_{l} \lambda(t) d t+r_{l} \delta(t) d t
$$

The total value of the accounts is the difference between the value of the strategy and the value of the purchased stocks, i.e., $\beta(t)+\lambda(t)+\delta(t)=V(S, t)-\alpha(t) S(t)$. In the classical Black-Scholes setting, this value would grow at the unique risk-free rate. However, in our model the lending rate determines the evolution of the cash and collateral account, while the borrowing rate determines the evolution of the debt account. Therefore, we must carefully segregate the positions into $i$ ) the collateral $M_{c}(t)$ required to be deposited in the cash account earning the lending rate, $i i)$ the quantity $V(S, t)-M_{c}(t)$ borrowed at the borrowing rate from the debt account to finance the posting of the margin, and finally iii) $\alpha(t) S(t)$ borrowed from the debt account to finance the stock purchase. 
Since the value of $M_{c}(t)$ is always greater than $V(S, t)$, the difference $V(S, t)-M_{c}(t)$ is negative and needs to be borrowed from the debt account. Summing up all positions in the debt and cash account and using the appropriate interest rates yields the following dynamics for the value of the accounts:

$$
d(\beta(t)+\lambda(t)+\delta(t))=\left(r_{l} M_{c}(t)-r_{b}\left(M_{c}(t)-V(S, t)\right)-r_{b} \alpha(t) S(t)\right) d t
$$

Since the value of the replicating strategy equals the value of the derivative, the option value must satisfy the PDE

$$
V_{t}^{\prime}(S, t)+\frac{1}{2} \sigma^{2} S^{2}(t) V_{s s}^{\prime \prime}(S, t)=r_{b} V(S, t)-\left(r_{b}-r_{l}\right) M_{c}(t)-\left(r_{b}-r_{d}\right) \alpha(t) S(t)
$$

which we can rewrite as

$$
V_{t}^{\prime}(S, t)+\left(r_{b}-r_{d}\right) S(t) V_{s}^{\prime}(S, t)+\frac{1}{2} \sigma^{2} S(t)^{2} V_{s s}^{\prime \prime}(S, t)=r_{b} V(S, t)-\left(r_{b}-r_{l}\right) M_{c}(t)
$$

with the boundary condition

$$
V(S, T)=(S(T)-K)^{+}
$$

The continuity of $M_{c}(t)$ allows us to make use of the Feynman-Kac Theorem to represent the solution to the PDE in (19) in terms of the following expectation: ${ }^{16}$

$$
V(S, t)=\underbrace{\mathbb{E}_{t}^{\mathbb{P}_{b}}\left[e^{-r_{b}(T-t)} V(S, T)\right]}_{(A)}+\underbrace{\mathbb{E}_{t}^{\mathbb{P}_{b}}\left[\int_{t}^{T} e^{-r_{b}(u-t)}\left(r_{b}-r_{l}\right) M_{c}(u) d u\right]}_{(B)} .
$$

We note that the expectation in equation (21) is taken under that pricing measure $\mathbb{P}_{b}$ for which the stock price discounted by $r_{b}-r_{d}$ is a martingale.

To replicate a put option, the investor has to short sell a certain amount of the underlying and invest it in the cash account. Hence we have three positions: i) the collateral $M_{p}(t)$ deposited in the cash account, $i i)$ the quantity $V(S, t)-M_{p}(t)$ borrowed to finance the required

\footnotetext{
${ }^{16}$ We remark that the solution to equation (21) is indeed the solution to the $\mathcal{M}^{+}$problem. It is the value of a self-financing strategy satisfying the collateral requirement of the option writers. Its payoff at time $T$ is equal to the payoff of the call option. Furthermore, no simultaneous borrowing and lending in the debt and cash account is involved in the replicating strategy. Therefore, the initial investment cost is minimized.
} 
margin, and iii) the short sell proceeds $\alpha(t) S(t)$ deposited in the cash account. ${ }^{17}$ The total growth of the cash, debt, and collateral account is then equal to

$$
d(\beta(t)+\lambda(t)+\delta(t))=\left[r_{l} M_{p}(t)-r_{b}\left(M_{p}(t)-V(S, t)\right)-r_{l} \alpha(t) S(t)\right] d t .
$$

Equating the replicating strategy value with the put option value $V(S, t)$ gives us the PDE for $V(S, t)$ :

$$
V_{t}^{\prime}(S, t)+\left(r_{l}-r_{d}\right) S(t) V_{s}^{\prime}(S, t)+\frac{1}{2} \sigma^{2} S^{2}(t) V_{s s}^{\prime \prime}(S, t)=r_{b} V(S, t)-\left(r_{b}-r_{l}\right) M_{p}(t),
$$

with the boundary condition

$$
V(S, T)=(K-S(T))^{+}
$$

When $M_{p}(t)$ is continuous, we can alternatively represent the PDE by ${ }^{18}$

$$
V(S, t)=\mathbb{E}_{t}^{\mathbb{P}_{l}}\left[e^{-r_{b}(T-t)} V(S, T)+\int_{t}^{T} e^{-r_{b}(u-t)}\left(r_{b}-r_{l}\right) M_{p}(u) d u\right]
$$

We note that for put options, the underlying has a drift term $r_{l}-r_{d}$ under the pricing measure $\mathbb{P}_{l}$, compared with $r_{b}-r_{d}$ for calls. According to the Feynman-Kac formula, the drift term of the underlying under the risk-neutral measure is determined by the coefficient of $\frac{\partial V(S, t)}{\partial S}$ in the PDE. For puts, the short sale proceeds are invested at $r_{l}$ while for calls, longing the underlying requires borrowing at $r_{b}$. Therefore, for puts and calls, different drift terms adjusting for dividends are applied to the underlying under the risk-neutral measure.

\section{B Options under the CBOE pricing rule}

We derive the call option price upper bound under the CBOE margin rule. The pricing formulas for put options can be computed similarly and are not given here. As described in

\footnotetext{
${ }^{17}$ Bergman (1995) even discusses the case when brokers collect the interest rates to compensate for their own monitoring costs. In such a case, the replicating costs for put options are even higher. However, as we only consider two rates in our model, we keep the assumption that short selling earns the lending rate.

${ }^{18}$ Given our assumption that short selling profits earn the lending rate, the solution given by equation (24) solves the $\mathcal{M}^{+}$for put options for the same reasons that we gave for calls.
} 
Section 2, the margin rule for call options in the CBOE is the piece-wise linear function

$$
M_{c}(t)= \begin{cases}a_{2} S(t)+V(S, t), & S(t) \leq \frac{1}{1+a_{1}-a_{2}} K \\ \left(1+a_{1}\right) S(t)-K+V(S, t), & \frac{1}{1+a_{1}-a_{2}} K<S(t) \leq K \\ a_{1} S(t)+V(S, t), & S(t)>K\end{cases}
$$

We can rewrite equation (19) to get

$\frac{\partial V(S, t)}{\partial t}+\left(r_{b}-r_{d}\right) S(t) \frac{\partial V(S, t)}{\partial S(t)}+\frac{1}{2} \sigma^{2} S(t)^{2} \frac{\partial^{2} V(S, t)}{\partial S(t)^{2}}=r_{l} V(S, t)-\left(r_{b}-r_{l}\right)\left(M_{c}(t)-V(S, t)\right)$.

Representing equation (25) as an expectation value, we obtain an alternative representation of equation (21):

$$
V(S, t)=\mathbb{E}_{t}^{\mathbb{P}_{b}}\left[e^{-r_{l}(T-t)} V(S, T)+\int_{t}^{T} e^{-r_{l}(u-t)}\left(r_{b}-r_{l}\right)\left(M_{c}(u)-V(u)\right) d u\right] .
$$

Plugging the margin function into equation (26) yields

$$
\begin{aligned}
V(S, t)= & \mathbb{E}_{t}^{\mathbb{P}_{b}}\left[e^{-r_{l}(T-t)} V(S, T)\right]+\mathbb{E}_{t}^{\mathbb{P}_{b}}\left[\int_{t}^{T} e^{-r_{l}(u-t)}\left(r_{b}-r_{l}\right) a_{2} S(u) \mathbf{1}_{\left\{S(u) \leq \frac{1}{1+a_{1}-a_{2}}\right.} K\right\} \\
& +\mathbb{E}_{t}^{\mathbb{P}_{b}}\left[\int_{t}^{T} e^{-r_{l}(u-t)}\left(r_{b}-r_{l}\right)\left(\left(1+a_{1}\right) S(u)-K\right) \mathbf{1}_{\left\{\frac{1}{1+a_{1}-a_{2}}\right.} K<S(u) \leq K\right\} \\
& +\mathbb{E}_{t}^{\mathbb{P}_{b}}\left[\int_{t}^{T} e^{-r_{l}(u-t)}\left(r_{b}-r_{l}\right) a_{1} S(u) \mathbf{1}_{\{S(u)>K\}} d u\right] \\
= & \mathbb{E}_{t}^{\mathbb{P}_{b}}\left[e^{-r_{l}(T-t)} V(S, T)\right]+a_{2}\left(r_{b}-r_{l}\right) \int_{t}^{T} e^{-r_{l}(u-t)} \mathbb{E}_{t}^{\mathbb{P}_{b}}\left[S(u) \mathbf{1}_{\left\{S(u) \leq \frac{1}{1+a_{1}-a_{2}}\right.} K\right\} \\
& \left.+\left(1+a_{1}\right)\left(r_{b}-r_{l}\right) \int_{t}^{T} e^{-r_{l}(u-t)} \mathbb{E}_{t}^{\mathbb{P}_{b}}\left[S(u) \mathbf{1}_{\left\{\frac{1}{1+a_{1}-a_{2}}\right.} K<S(u) \leq K\right\}\right] d u \\
& \left.-\left(r_{b}-r_{l}\right) K \int_{t}^{T} e^{-r_{l}(u-t)} \mathbb{E}_{t}^{\mathbb{P}_{b}}\left[\mathbf{1}_{\left\{\frac{1}{1+a_{1}-a_{2}}\right.} K<S(u) \leq K\right\}\right] d u \\
& +a_{1}\left(r_{b}-r_{l}\right) \int_{t}^{T} e^{-r_{l}(u-t)} \mathbb{E}_{t}^{\mathbb{P}_{b}}\left[S(u) \mathbf{1}_{\{S(u)>K\}}\right] d u .
\end{aligned}
$$

The first term is just the Black-Scholes price under a different measure. Under the probability measure $\mathbb{P}_{b}$, we have $d S(t) / S(t)=\left(r_{b}-r_{d}\right) d t+\sigma d W^{b}(t)$. Moreover, $W^{b}(u)-W^{b}(t)$ is a zeromean normal variable with variance $u-t$. The conditional expectations can be computed using the lognormal distribution of $S(t)$ and are omitted for brevity. Interchanging these 
expectations into the call option value yields

$$
\begin{aligned}
V_{c}(t)= & S(t) e^{\left(r_{b}-r_{d}-r_{l}\right)(T-t)} N\left(d_{1}(T, t)\right)-K e^{-r_{l}(T-t)} N\left(d_{2}(T, t)\right) \\
& +\left(r_{b}-r_{l}\right) S(t) \int_{t}^{T} e^{\left(r_{b}-r_{d}-r_{l}\right)(u-t)}\left(a_{2} N\left(-d_{1}^{*}(u, t)\right)+a_{1} N\left(d_{1}(u, t)\right)\right) d u \\
& +\left(1+a_{1}\right)\left(r_{b}-r_{l}\right) S(t) \int_{t}^{T} e^{\left(r_{b}-r_{d}-r_{l}\right)(u-t)}\left(N\left(d_{1}^{*}(u, t)\right)-N\left(d_{1}(u, t)\right)\right) d u \\
& -\left(r_{b}-r_{l}\right) K \int_{t}^{T} e^{-r_{l}(u-t)}\left(N\left(d_{2}^{*}(u, t)\right)-N\left(d_{2}(u, t)\right)\right) d u
\end{aligned}
$$

where $d_{1,2}(u, t)$ and $d_{1,2}^{*}(u, t)$ are defined in equation (9). 


\begin{tabular}{lcccccc} 
& $\exp \left(\Pi_{0}\right)$ & $\Pi$ & Adjusted $R^{2}$ & $\exp \left(\Pi_{0}\right)$ & $\Pi$ & Adjusted $R^{2}$ \\
\hline Short-term & 0.184 & -3.381 & 0.730 & 0.191 & -2.923 & 0.712 \\
& $(-62.210)$ & $(-32.104)$ & & $(-59.085)$ & $(-29.852)$ & \\
Medium-term & 0.186 & -2.105 & 0.855 & 0.192 & -1.957 & 0.920 \\
& $(-68.913)$ & $(-37.549)$ & & $(-67.193)$ & $(-43.247)$ & \\
\hline
\end{tabular}

Table 1: Regression results for obtaining the empirical slopes. The table displays the results for the regression (12) of implied volatility on moneyness for call and put options with $t$-statistics in parentheses. We ran the regression for each week of our sample period from January 2002 to August 2010 for a total of 449 weeks. The term $\exp \left(\Pi_{0}\right)$ represents the implied volatility for at-the-money options. The reported coefficients and adjusted $R^{2}$ are time averages over all 449 weeks. The $t$ statistics are the time-series average of the weekly estimates divided by the standard deviation of the average adjusted for first-order autocorrelation (BKM (2003)). Short-term options are those with maturities less than 60 days. Medium-term options have expirations between 60 to 150 days. 


\begin{tabular}{|c|c|c|c|c|c|c|c|c|}
\hline & \multicolumn{4}{|c|}{ Short-term } & \multicolumn{4}{|c|}{ Medium-term } \\
\hline & $\begin{array}{c}\text { only } \\
\text { lag }\end{array}$ & $\begin{array}{c}\text { strategy } \\
\text { margin }\end{array}$ & $\begin{array}{c}\text { portfolio } \\
\text { short }\end{array}$ & $\begin{array}{c}\text { minimum } \\
\text { portfolio }\end{array}$ & $\begin{array}{c}\text { only } \\
\text { lag }\end{array}$ & $\begin{array}{c}\text { strategy } \\
\text { margin }\end{array}$ & $\begin{array}{c}\text { portfolio } \\
\text { short }\end{array}$ & $\begin{array}{c}\text { minimum } \\
\text { portfolio }\end{array}$ \\
\hline & \multicolumn{8}{|c|}{ Panel A: Call options } \\
\hline$\Delta \Pi_{t}^{\text {model }}$ & & $\begin{array}{c}3.253 \\
(8.844)\end{array}$ & $\begin{array}{c}2.569 \\
(9.540)\end{array}$ & $\begin{array}{c}2.592 \\
(9.562)\end{array}$ & & $\begin{array}{c}1.763 \\
(13.704)\end{array}$ & $\begin{array}{c}1.536 \\
(15.304)\end{array}$ & $\begin{array}{c}1.551 \\
(15.341)\end{array}$ \\
\hline$\Delta \Pi_{t-1}$ & $\begin{array}{c}-0.238 \\
(-4.968)\end{array}$ & $\begin{array}{c}-0.225 \\
(-5.983)\end{array}$ & $\begin{array}{c}-0.222 \\
(-6.170)\end{array}$ & $\begin{array}{c}-0.222 \\
(-6.178)\end{array}$ & $\begin{array}{c}-0.286 \\
(-6.677)\end{array}$ & $\begin{array}{c}-0.295 \\
(-6.469)\end{array}$ & $\begin{array}{c}-0.288 \\
(-6.450)\end{array}$ & $\begin{array}{c}-0.288 \\
(-6.454)\end{array}$ \\
\hline \multirow[t]{2}{*}{ Adjusted $R^{2}$} & 0.054 & 0.227 & 0.257 & 0.258 & 0.080 & 0.408 & 0.421 & 0.421 \\
\hline & \multicolumn{8}{|c|}{ Panel B: Put options } \\
\hline$\Delta \Pi_{t}^{\text {model }}$ & & $\begin{array}{c}6.603 \\
(9.168)\end{array}$ & $\begin{array}{l}10.679 \\
(5.244)\end{array}$ & $\begin{array}{c}5.066 \\
(7.617)\end{array}$ & & $\begin{array}{c}3.988 \\
(13.052)\end{array}$ & $\begin{array}{c}8.409 \\
(9.756)\end{array}$ & $\begin{array}{c}4.128 \\
(12.600)\end{array}$ \\
\hline$\Delta \Pi_{t-1}$ & $\begin{array}{c}-0.312 \\
(-5.201)\end{array}$ & $\begin{array}{c}-0.317 \\
(-6.009)\end{array}$ & $\begin{array}{c}-0.306 \\
(-5.578)\end{array}$ & $\begin{array}{c}-0.314 \\
(-5.983)\end{array}$ & $\begin{array}{c}-0.195 \\
(-7.131)\end{array}$ & $\begin{array}{c}-0.218 \\
(-6.251)\end{array}$ & $\begin{array}{c}-0.163 \\
(-5.543)\end{array}$ & $\begin{array}{c}-0.165 \\
(-5.385)\end{array}$ \\
\hline Adjusted $R^{2}$ & 0.095 & 0.238 & 0.200 & 0.238 & 0.036 & 0.463 & 0.277 & 0.357 \\
\hline
\end{tabular}

Table 2: Regression results for changes of empirical IV slopes on changes of model-implied slopes. The table reports the estimated coefficients from regressing the differences of empirical IV slope on the lagged differences and also on differences of model-implied slopes. We give corresponding $t$-statistics in parentheses. Panel A shows the results for both short-term and medium-term call options. Panel B reports the results for short-term and medium-term put options. For each option category, we report the results of the regression using the lagged dependent variable alone and also for the combined regression using three margin rules, namely strategy margins for a naked short sale, the portfolio margins for a naked short sale, and minimum portfolio margin requirements. The standard errors used to compute the $t$-statistics are the Newey-West estimates with a lag length of 5 . 


\begin{tabular}{|c|c|c|c|c|c|c|c|c|}
\hline & \multicolumn{4}{|c|}{ Short-term } & \multicolumn{4}{|c|}{ Medium-term } \\
\hline & $\begin{array}{c}\text { only } \\
\text { controls }\end{array}$ & $\begin{array}{c}\text { strategy } \\
\text { margin }\end{array}$ & $\begin{array}{c}\text { portfolio } \\
\text { short }\end{array}$ & $\begin{array}{l}\text { minimum } \\
\text { portfolio }\end{array}$ & $\begin{array}{c}\text { only } \\
\text { controls }\end{array}$ & $\begin{array}{c}\text { strategy } \\
\text { margin }\end{array}$ & $\begin{array}{c}\text { portfolio } \\
\text { short }\end{array}$ & $\begin{array}{l}\text { minimum } \\
\text { portfolio }\end{array}$ \\
\hline & \multicolumn{8}{|c|}{ Panel A: Call options } \\
\hline \multirow[t]{2}{*}{$\Delta \Pi_{t}^{\text {model }}$} & & 3.276 & 2.586 & 2.609 & & 1.706 & 1.496 & 1.510 \\
\hline & & $(8.839)$ & $(9.518)$ & $(9.541)$ & & $(12.017)$ & $(13.597)$ & $(13.638)$ \\
\hline \multirow[t]{2}{*}{$\Delta$ Skewness $_{t}$} & 0.108 & 0.177 & 0.194 & 0.194 & -0.222 & -0.034 & 0.012 & 0.014 \\
\hline & $(0.371)$ & $(0.695)$ & $(0.788)$ & $(0.790)$ & $(-1.585)$ & $(-0.300)$ & $(0.109)$ & $(0.128)$ \\
\hline \multirow{2}{*}{$\Delta$ Kurtosis $_{t}$} & 0.007 & 0.023 & 0.025 & 0.177 & -0.078 & -0.019 & -0.012 & -0.011 \\
\hline & $(0.203)$ & $(0.743)$ & $(0.824)$ & $(0.824)$ & $(-2.544)$ & $(-0.793)$ & $(-0.526)$ & $(-0.514)$ \\
\hline \multirow[t]{2}{*}{$\Delta \Pi_{t-1}$} & -0.237 & -0.227 & -0.224 & -0.224 & -0.300 & -0.300 & -0.291 & -0.291 \\
\hline & $(-4.850)$ & $(-5.955)$ & $(-6.155)$ & $(-6.163)$ & $(-6.455)$ & $(-6.258)$ & $(-6.205)$ & $(-6.205)$ \\
\hline \multirow[t]{2}{*}{ Adjusted $R^{2}$} & 0.051 & 0.225 & 0.255 & 0.207 & 0.126 & 0.409 & 0.422 & 0.422 \\
\hline & \multicolumn{8}{|c|}{ Panel B: Put options } \\
\hline \multirow[t]{2}{*}{$\Delta \Pi_{t}^{\text {model }}$} & & 6.684 & 10.723 & 5.106 & & 3.889 & 7.907 & 3.955 \\
\hline & & $(9.056)$ & $(5.295)$ & $(7.641)$ & & $(11.549)$ & $(8.235)$ & $(12.178)$ \\
\hline \multirow[t]{2}{*}{$\Delta$ Skewness $_{t}$} & 0.228 & 0.344 & 0.270 & 0.317 & -0.300 & -0.055 & -0.101 & -0.070 \\
\hline & $(0.942)$ & $(1.725)$ & $(1.409)$ & $(1.651)$ & $(-0.168)$ & $(-0.561)$ & $(-0.851)$ & $(-0.644)$ \\
\hline \multirow[t]{2}{*}{$\Delta$ Kurtosis $_{t}$} & 0.021 & 0.038 & 0.025 & 0.034 & -0.075 & -0.016 & -0.033 & -0.025 \\
\hline & $(0.869)$ & $(1.839)$ & $(1.248)$ & $(1.695)$ & $(-3.139)$ & $(-0.968)$ & $(-1.453)$ & $(-1.222)$ \\
\hline \multirow[t]{2}{*}{$\Delta \Pi_{t-1}$} & -0.313 & -0.314 & -0.307 & -0.312 & -0.172 & -0.221 & -0.172 & -0.171 \\
\hline & $(-5.199)$ & $(-5.894)$ & $(-5.575)$ & $(-5.885)$ & $(-5.667)$ & $(-6.243)$ & $(-5.667)$ & $(-5.566)$ \\
\hline Adjusted $R^{2}$ & 0.093 & 0.239 & 0.199 & 0.239 & 0.089 & 0.463 & 0.288 & 0.335 \\
\hline
\end{tabular}

Table 3: Regression results for changes of empirical IV slopes on changes of risk-neutral parameters. The table reports the estimated coefficients for regressions explaining the difference of slopes using the difference of risk-neutral parameters. Panel A shows the results for call options and Panel B for put options. We analyze short-term and medium-term options separately. Column only controls shows the regression where only control variables are employed. We also run combined regressions using implied slopes. Results for regressions incorporating slopes derived from each type of margin rule are shown in the column labeled according to the margin rule. The standard errors used to compute the $t$-statistics are the Newey-West estimates with a lag length of 5 . 


\begin{tabular}{|c|c|c|c|c|c|c|c|c|}
\hline & \multicolumn{4}{|c|}{ Short-term } & \multicolumn{4}{|c|}{ Medium-term } \\
\hline & $\begin{array}{c}\text { only } \\
\text { controls }\end{array}$ & $\begin{array}{c}\text { strategy } \\
\text { margin }\end{array}$ & $\begin{array}{c}\text { portfolio } \\
\text { short }\end{array}$ & $\begin{array}{l}\text { minimum } \\
\text { portfolio }\end{array}$ & $\begin{array}{c}\text { only } \\
\text { controls }\end{array}$ & $\begin{array}{c}\text { strategy } \\
\text { margin }\end{array}$ & $\begin{array}{c}\text { portfolio } \\
\text { short }\end{array}$ & $\begin{array}{l}\text { minimum } \\
\text { portfolio }\end{array}$ \\
\hline & \multicolumn{8}{|c|}{ Panel A: Call options } \\
\hline$\Delta \Pi_{t}^{\text {model }}$ & & $\begin{array}{c}3.374 \\
(8.709)\end{array}$ & $\begin{array}{c}2.627 \\
(9.080)\end{array}$ & $\begin{array}{c}2.648 \\
(9.093)\end{array}$ & & $\begin{array}{c}1.725 \\
(10.224)\end{array}$ & $\begin{array}{c}1.484 \\
(13.705)\end{array}$ & $\begin{array}{c}1.495 \\
(13.752)\end{array}$ \\
\hline$\Delta$ LiborOIS $_{t}$ & $\begin{array}{c}-0.207 \\
(-0.714)\end{array}$ & $\begin{array}{c}0.524 \\
(1.929)\end{array}$ & $\begin{array}{c}0.536 \\
(1.210)\end{array}$ & $\begin{array}{c}0.530 \\
(2.138)\end{array}$ & $\begin{array}{c}-0.081 \\
(-0.649)\end{array}$ & $\begin{array}{c}0.261 \\
(1.676)\end{array}$ & $\begin{array}{c}0.231 \\
(2.129)\end{array}$ & $\begin{array}{c}0.226 \\
(2.114)\end{array}$ \\
\hline$\Delta \mathrm{VIX}_{t}$ & $\begin{array}{c}0.075 \\
(3.500)\end{array}$ & $\begin{array}{c}-0.023 \\
(-1.837)\end{array}$ & $\begin{array}{c}-0.021 \\
(-1.070)\end{array}$ & $\begin{array}{c}-0.021 \\
(-1.673)\end{array}$ & $\begin{array}{c}0.044 \\
(4.472)\end{array}$ & $\begin{array}{c}0.004 \\
(0.941)\end{array}$ & $\begin{array}{c}0.007 \\
(1.584)\end{array}$ & $\begin{array}{c}0.007 \\
(1.681)\end{array}$ \\
\hline$\Delta$ IndexReturn $_{t}$ & $\begin{array}{c}-0.277 \\
(-0.289)\end{array}$ & $\begin{array}{c}-0.428 \\
(-0.452)\end{array}$ & $\begin{array}{c}-0.522 \\
(-0.409)\end{array}$ & $\begin{array}{c}-0.525 \\
(-0.555)\end{array}$ & $\begin{array}{c}0.020 \\
(0.060)\end{array}$ & $\begin{array}{c}-0.154 \\
(-0.469)\end{array}$ & $\begin{array}{c}-0.172 \\
(-0.522)\end{array}$ & $\begin{array}{c}-0.173 \\
(-0.527)\end{array}$ \\
\hline$\Delta$ RelativeDemand $_{t}$ & $\begin{array}{c}-0.356 \\
(-2.700)\end{array}$ & $\begin{array}{c}-0.273 \\
(-2.215)\end{array}$ & $\begin{array}{c}-0.237 \\
(-2.285)\end{array}$ & $\begin{array}{c}-0.237 \\
(-1.954)\end{array}$ & $\begin{array}{c}0.004 \\
(0.075)\end{array}$ & $\begin{array}{c}0.010 \\
(0.227)\end{array}$ & $\begin{array}{c}0.003 \\
(0.089)\end{array}$ & $\begin{array}{c}0.003 \\
(0.084)\end{array}$ \\
\hline$\Delta \Pi_{t-1}$ & $\begin{array}{c}-0.221 \\
(-4.956)\end{array}$ & $\begin{array}{c}-0.218 \\
(-5.754)\end{array}$ & $\begin{array}{c}-0.217 \\
(-5.959)\end{array}$ & $\begin{array}{c}-0.217 \\
(-5.307)\end{array}$ & $\begin{array}{c}-0.286 \\
(-7.301)\end{array}$ & $\begin{array}{c}-0.299 \\
(-6.720)\end{array}$ & $\begin{array}{c}-0.291 \\
(-6.712)\end{array}$ & $\begin{array}{c}-0.291 \\
(-6.717)\end{array}$ \\
\hline \multirow[t]{2}{*}{ Adjusted $R^{2}$} & 0.116 & 0.235 & 0.262 & 0.263 & 0.159 & 0.409 & 0.424 & 0.424 \\
\hline & \multicolumn{8}{|c|}{ Panel B: Put options } \\
\hline$\Delta \Pi_{t}^{\text {model }}$ & & $\begin{array}{c}5.995 \\
(7.229)\end{array}$ & $\begin{array}{c}9.625 \\
(4.619)\end{array}$ & $\begin{array}{c}4.600 \\
(6.137)\end{array}$ & & $\begin{array}{c}3.755 \\
(11.895)\end{array}$ & $\begin{array}{c}8.276 \\
(9.865)\end{array}$ & $\begin{array}{c}3.903 \\
(11.962)\end{array}$ \\
\hline$\Delta$ LiborOIS $_{t}$ & $\begin{array}{c}-0.262 \\
(-0.808)\end{array}$ & $\begin{array}{c}0.121 \\
(0.505)\end{array}$ & $\begin{array}{c}-0.201 \\
(-0.806)\end{array}$ & $\begin{array}{c}0.050 \\
(0.238)\end{array}$ & $\begin{array}{c}-0.112 \\
(-0.997)\end{array}$ & $\begin{array}{c}0.015 \\
(0.230)\end{array}$ & $\begin{array}{c}-0.142 \\
(-1.357)\end{array}$ & $\begin{array}{c}-0.064 \\
(-0.747)\end{array}$ \\
\hline$\Delta \mathrm{VIX}_{t}$ & $\begin{array}{c}0.083 \\
(3.430)\end{array}$ & $\begin{array}{c}0.026 \\
(2.282)\end{array}$ & $\begin{array}{c}0.062 \\
(3.516)\end{array}$ & $\begin{array}{c}0.041 \\
(3.054)\end{array}$ & $\begin{array}{c}0.036 \\
(4.298)\end{array}$ & $\begin{array}{c}0.017 \\
(3.627)\end{array}$ & $\begin{array}{c}0.034 \\
(4.538)\end{array}$ & $\begin{array}{c}0.028 \\
(4.200)\end{array}$ \\
\hline$\Delta$ IndexReturn $_{t}$ & $\begin{array}{c}-0.986 \\
(-1.156)\end{array}$ & $\begin{array}{c}-1.242 \\
(-1.563)\end{array}$ & $\begin{array}{c}-1.427 \\
(-1.749)\end{array}$ & $\begin{array}{c}-1.448 \\
(-1.732)\end{array}$ & $\begin{array}{c}0.040 \\
(0.128)\end{array}$ & $\begin{array}{c}-0.159 \\
(-0.522)\end{array}$ & $\begin{array}{c}-0.019 \\
(-0.064)\end{array}$ & $\begin{array}{c}-0.069 \\
(-0.221)\end{array}$ \\
\hline$\Delta$ RelativeDemand $_{t}$ & $\begin{array}{c}-0.029 \\
(-0.231)\end{array}$ & $\begin{array}{c}0.063 \\
(0.535)\end{array}$ & $\begin{array}{c}0.051 \\
(0.431)\end{array}$ & $\begin{array}{c}0.089 \\
(0.737)\end{array}$ & $\begin{array}{c}0.023 \\
(0.584)\end{array}$ & $\begin{array}{c}0.025 \\
(0.883)\end{array}$ & $\begin{array}{c}0.006 \\
(0.201)\end{array}$ & $\begin{array}{c}0.006 \\
(0.220)\end{array}$ \\
\hline$\Delta \Pi_{t-1}$ & $\begin{array}{c}-0.322 \\
(-5.634)\end{array}$ & $\begin{array}{c}-0.323 \\
(-6.246)\end{array}$ & $\begin{array}{c}-0.316 \\
(-5.952)\end{array}$ & $\begin{array}{c}-0.322 \\
(-6.316)\end{array}$ & $\begin{array}{c}-0.204 \\
(-7.160)\end{array}$ & $\begin{array}{c}-0.225 \\
(-6.239)\end{array}$ & $\begin{array}{c}-0.170 \\
(-5.940)\end{array}$ & $\begin{array}{c}-0.174 \\
(-5.574)\end{array}$ \\
\hline Adjusted $R^{2}$ & 0.149 & 0.241 & 0.230 & 0.252 & 0.127 & 0.484 & 0.362 & 0.412 \\
\hline
\end{tabular}

Table 4: Regression results for changes of empirical IV slopes. The table shows the estimated coefficients from regressing the changes of empirical slopes on changes of the second set control variables. Panel A reports the results for calls, Panel B reports the results for puts. We analyze short-term and medium-term options separately. Column only controls shows the regression where only control variables are employed. We also run combined regressions using implied slopes. Results for regressions using also slopes derived from each type of margin rule are shown in the column labeled according to the margin rule. The standard errors used to compute the $t$-statistics are the Newey-West estimates with a lag length of 5 . 